\title{
Model Predictive Glycaemic Regulation in Critical Illness using Insulin and Nutrition Input: a Pilot Study
}

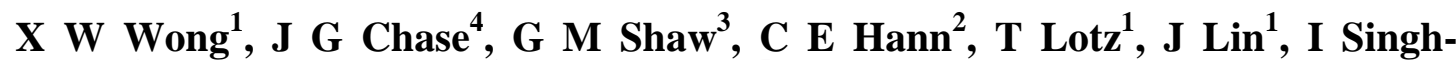 \\ Levett $^{1}$, L J Hollingsworth ${ }^{1}$, O S W Wong ${ }^{5}$ and S Andreassen ${ }^{6}$
}

1 Department of Mechanical Engineering, College of Engineering, University of Canterbury, Christchurch, New Zealand

2 PhD, Department of Mechanical Engineering, University of Canterbury, Christchurch, New Zealand

MbChB, FJFICM, Department of Intensive Care, Christchurch Hospital, Christchurch School of Medicine and Health Science, University of Otago, Dunedin, Christchurch

$4 \quad$ PhD, Department of Mechanical Engineering, University of Canterbury, Christchurch, Christchurch School of Medicine and Health Science, University of Otago, Dunedin, New Zealand

5 Christchurch School of Medicine and Health Science, University of Otago, Dunedin, New Zealand

$6 \quad$ Reader, Centre for Model-based Medical Decision Support, University of Aalborg, Aalborg, Denmark

Work performed at:

Department of Intensive Care, Christchurch Hospital

Department of Mechanical Engineering, University of Canterbury

Christchurch School of Medicine and Health Sciences, University of Otago

Address for Reprints: Reprints available from

Dr J. Geoffrey Chase

Department of Mechanical Engineering

University of Canterbury

Private Bag 4800

Christchurch

New Zealand

geoff.chase@canterbury.ac.nz

Financial Support:

Canterbury Intensive Care Research and Education Trust

Tertiary Education Commission Te Amorangi Matauranga Matua

Keywords:

Critical Care, Hyperglycemia, Retrospective Studies, ICU, Blood Glucose, Insulin 


\section{INTRODUCTION}

Critically ill patients often experience stress-induced hyperglycaemia and high insulin resistance, even with no prior diabetes [1-4]. The increased counter-regulatory hormone and cytokine response stimulates endogenous glucose production and increases effective insulin resistance. Absolute and relative insulin deficiency, and drug and steroid use are further causes. Studies also indicate that high glucose content nutritional support regimes result in excess glucose [5-7]. More recently, reductions in enteral nutrition [8], and the use of dextrose as a diluent in intravenous medication [9], resulted in reductions in glycaemia, and the alleviation of the counterregulatory response $[3,4,10,11]$.

Although hyperglycaemia can be a marker of severity of illness, it also worsens outcomes, leading to an increased risk of further complications such as severe infection [12], myocardial infarction [1], and critical illnesses such as polyneuropathy and multiple-organ failure [2]. There is also evidence of significant reductions in need for dialysis, bacteremia and number of blood transfusions with aggressive blood glucose control [13]. van den Berghe [2, 14] showed that tight glucose control to less than $6.1 \mathrm{mmol} / \mathrm{L}$ reduced cardiac ICU patient mortality by up to $45 \%$ while Krinsley $[15,16]$ showed a $17-29 \%$ total reduction in mortality over a wider ICU population with a higher glucose limit of $7.75 \mathrm{mmol} / \mathrm{L}$.

Short-term, adaptive model-based protocols for insulin-mediated glucose control have been developed utilising integral-based identification of time-varying patient specific parameters $[17,18]$. These insulin-based protocols are suited for clinical use, but 
have limitations. More specifically, high effective insulin resistance due to stress of condition and increased counter-regulatory dynamics can result in hyperglycaemia regardless of hyperinsulinaemia $[17,18]$. Metabolic clearance rate is not stimulated with hyperinsulinaemia under conditions of stress, as endogenous glucose production is, which compounds hyperglycaemia $[19,20]$. Insulin effect can also saturate at the supraphysiological insulin concentrations that exist under aggressive insulin therapy [18, 21-25], limiting the achievable glycaemic reduction using insulin alone if effective insulin resistance is high.

As a result, exogenous nutritional inputs must be reduced to effect a reduction in hyperglycaemia. In critical care, research by Patino et al. [5] with lower glucose nutrition alone has seen significant reductions in average blood glucose levels. Additionally, Patino et al. found poorer clinical outcome of hyperdynamic cardiorespiratory repercussion, high $\mathrm{CO}_{2}$ production, and hepatic steatosis with high caloric loads, and no suppression of excessive endogenous glucose production with hypercaloric TPN. Hence, reduced glucose nutrition combined with insulin administration can act to control both sides (input and removal) of the glucose balance.

This paper develops and presents a pilot study of a long-term controller for safe regulation of glycaemia under elevated insulin resistance and glucose intolerance in critically ill patients by modulating enteral nutrition inputs in addition to conventional basal-bolus intravenous insulin therapy. Clinical proof-of-concept pilot trials of the algorithm are performed which show the algorithm adaptability to time-varying intraas well as inter-patient variability in condition while requiring relatively infrequent 
glucose measurement. This research is a step towards randomized, comparative cohort studies of clinical outcomes using the developed protocol.

Previous blood glucose control research includes controlled experiments in insulin infusion by Hovorka et al. [26], Chee et al. [27], and Chase et al. [18, 28]. Adaptive bolus-based control using insulin-alone by Chase et al. [18], is the basis of this work. The primary difference in this research is the improvement in control under elevated insulin resistance by modulation of nutritional support in addition to insulin input.

\section{METHODS}

\section{$2.1 \quad$ System model}

Tight blood glucose control requires a patient-specific glucose-insulin regulatory system model that captures the fundamental dynamics. Chase et al. $[17,18]$ used an extended system model that captured rate of insulin utilisation, insulin losses and saturation dynamics, and is also used in this study:

$$
\begin{aligned}
& \dot{G}=-p_{G} G-S_{I}\left(G+G_{E}\right) \frac{Q}{1+\alpha_{G} Q}+P(t) \\
& \dot{Q}=-k I+k Q \\
& \dot{I}=-\frac{n I}{1+\alpha_{I} I}+\frac{u_{e x}}{V}
\end{aligned}
$$

The symbols $G(t)[\mathrm{mmol} / \mathrm{L}]$ and $I[\mathrm{mU} / \mathrm{L}]$ denote the plasma glucose above an equilibrium level, $G_{E}[\mathrm{mmol} / \mathrm{L}]$, and the plasma insulin resulting from exogenous 
insulin input, respectively. The insulin concentration at the interstitial sites is represented by $Q$ [mU/L], with $k$ [1/min] accounting for the effective life of insulin in the compartment. $I[\mathrm{mU} / \mathrm{L}]$ is the plasma insulin concentration with $n[1 / \mathrm{min}]$, the first order decay rate from plasma. Patient endogenous glucose clearance and insulin sensitivity are $p_{G}[1 / \mathrm{min}]$ and $S_{I}[\mathrm{~L} /(\mathrm{mU} \cdot \mathrm{min})]$, respectively. The parameter $V[\mathrm{~L}]$ is the insulin distribution volume. Total plasma glucose input is expressed as $P(t)$ [mmol/(L.min)], and insulin input, $u_{e x}(t)[\mathrm{mU} / \mathrm{min}]$. Michaelis-Menten functions are used to model saturation, with parameter $\alpha_{I}[\mathrm{~L} / \mathrm{mU}]$ for plasma insulin disappearance, and $\alpha_{G}[\mathrm{~L} / \mathrm{mU}]$ for insulin-dependent glucose clearance or insulin effect $[17,18]$.

For this study, $k, n, \alpha_{G}, \alpha_{I}$ and $V$ were initially set to generic population values [18, 29]. During the clinical trials, $k, \alpha_{G}$ and $V$ were tuned to match patient dynamics while the glucose distribution volume was taken as $0.19 \mathrm{~L} / \mathrm{kg}$ of the patient total body mass [30-32]. The parameters $n$ and $\alpha_{I}$ were set to $0.16 \mathrm{~min}^{-1}$ and $0.0017 \mathrm{~L} / \mathrm{mU}$ [18], which is consistent across many studies e.g. [33-36]. The parameter $\alpha_{G}=1 / 65 \mathrm{~L} / \mathrm{mU}$ is an initial conservative choice for likelier underestimation of saturation [18, 21, 37] while $k$ was set to $0.0198 \mathrm{~min}^{-1}$ for an effective insulin half-life of $35 \mathrm{~min}$ in the interstitial sites [18].

In Equation (1), $G_{E}$ represents the patient-specific equilibrium state under constant feed, insulin infusion, insulin sensitivity and glucose clearance. Due to ongoing exogenous insulin infusion and variable nutritional inputs, an instantaneous equilibrium blood glucose level can be difficult to determine [17]. Hence, the current glucose value is used to update $G_{E}$ every two hours after a feed rate modulation. 
Equation (3) does not include endogenous insulin or glucose production. Critically ill patients are also often hyperinsulinaemic. Although endogenous insulin production rate is fairly consistent across the non-critically ill population [35, 38-40], its value can be highly variable and difficult to obtain quickly in critical care. However, endogenous insulin production is suppressed with significant exogenous insulin administration [32, 35, 41]. In addition, endogenous glucose production is known to be unsuppressed by insulin or glycaemia under conditions of stress [19, 20]. Hence, in this study, an unknown glucose supply is assumed and its effect is lumped in the time-varying parameter, $p_{G}$ while endogenous insulin production is assumed to be suppressed to a steady constant value under significant exogenous insulin input. Any deviation from these assumptions will result in a scaling in the values of $S_{I}$, if $p_{G}$ is held constant, and the effect on control should be minimal.

\subsection{Variable enteral nutrition model}

Chase et al. [18] modelled the constant enteral feed rate in their trials, $P(t)$, as a constant value. In this study, stepwise enteral mixed meal glucose fluxes are employed for control and modelled using exponential functions.

$$
P\left(t_{i}<t<t_{i+1}\right)=\bar{P}_{i+1}+\left(P\left(t_{i}\right)-\bar{P}_{i+1}\right) e^{-k_{p d}\left(t-t_{i}\right)} \text { where } \bar{P}_{i+1}<P\left(t_{i}\right)
$$

$$
P\left(t_{i}<t<t_{i+1}\right)=\bar{P}_{i+1}+\left(P\left(t_{i}\right)-\bar{P}_{i+1}\right) e^{-k_{p r}\left(t-t_{i}\right)} \text { where } \bar{P}_{i+1}>P\left(t_{i}\right)
$$


where $k_{p r}$ and $k_{p d}(1 / \mathrm{min})$ are the effective half lives of glucose transport from gut to plasma for both increasing $\left(k_{p r}\right)$ and decreasing $\left(k_{p d}\right)$ feed rates respectively, and $\bar{P}_{i}$ and $\bar{P}_{i+1}$ are the steps in enteral glucose feed rates.

Many studies have investigated glucose kinetics under non-steady conditions using tracers. Postprandial glucose kinetics are characterised by suppression of hepatic glucose output in both an oral glucose load (OGL) and continuous feeding approach [30, 42, 43]. Glucose disposal rate is largely unaffected [30, 42, 44]. Time periods for glucose absorption range from 120 to 240min [43-46] for an OGL with a peak in total glucose at $\sim 40$ mins [43]. Slower absorption was evidenced in a mixed meal formulation [47], and [30] showed that mixed-meal postprandial GRa increased progressively until near steady-state between 230 and 270 minutes.

Oral glucose undergoes rapid intestinal absorption and a high percentage passes through the liver to appear systemically, sustaining a low first-pass splanchnic uptake [43]. Whether such dynamics are applicable to non-steady-state enteral glucose infusions where variations in glucose load are much smaller is unknown, although [43] showed no discernible difference in systemic oral glucose appearances with a half-sized oral glucose load. Any incretin effect present is likely to be minimal as enteral feeds are administered long-term and are held in steady state for at least $2 \mathrm{~h}$ before the commencement of the trial.

Model-based methods of calculating glucose fluxes [43, 48, 49] using tracer concentrations are limiting and unsuitable for real-time clinical application. Hence, different exponential rates for total GRa rise $\left(k_{p r}\right)$ and decay $\left(k_{p d}\right)$ can be used to 
model transient hepatic glucose output and first-pass splanchnic uptake in non-steady feeding. Impaired uptake of glucose in diabetes and stress-induced hyperglycaemia [30, 42, 50-53] imply a slow decay rate in total GRa following nutritional feed reduction. Conversely, rapid intestinal absorption implies a rapid rise rate in total GRa [43]. Therefore, the values of $k_{p r}$ and $k_{p d}$ are set to $0.0347 \mathrm{~min}^{-1}$ and $0.0068 \mathrm{~min}^{-1}$ from data in the literature, corresponding to half-lives of 20 and 100mins. Figure 1 shows the modelled total nutritional input, $P(t)$, following enteral feed rate variations using $k_{p r}=0.0347 \mathrm{~min}^{-1}$ and $k_{p d}=0.0068 \mathrm{~min}^{-1}$. Note that this model is only designed to capture the first order dynamics of glucose appearance as a function of enteral feed. Hence Equations (4)-(5) effectively assumes a linear 2-compartment model.

While the digestion process prior to absorption is highly variable, such variability is minimised by several means. Firstly, the mixed meal formulation is standardised. Also, an exclusion criterion of abnormal gastric emptying rates as determined from residual gastric aspirates (Section 3.1 Patient Cohort) is placed on prospective patients for the study. Lastly, enteral feed fluxes are calculated based on the rapidly absorbed glucose caloric content, not protein- and fat-derived calories. These steps reinforce the presumption that only the glucose is absorbed in the first-pass into plasma even though the protein and fat content contribute to the gluconeogenic input into plasma at some later time. Note also that the 2-compartment model mimics many standard mixed meal models used [54] in analysing diabetic responses. 


\subsection{Parameter fitting and prediction}

Prior to resolving a bolus size and feed rate to achieve a target, $S_{I}$ must be fitted. The parameter fitting is described in $[17,18]$. The main difference is the assumption of $p_{G}$ $=0.01$, a value found in [17] to be relatively constant and insensitive across a diverse ICU patient cohort. The fitted insulin sensitivity, $S_{I}$, is used to predict the blood glucose response in the following hour. The insulin sensitivity in the following hour $\left(\bar{S}_{\mathrm{I}_{(i+1)}}\right)$ is estimated as a function of previously fitted insulin sensitivity values.

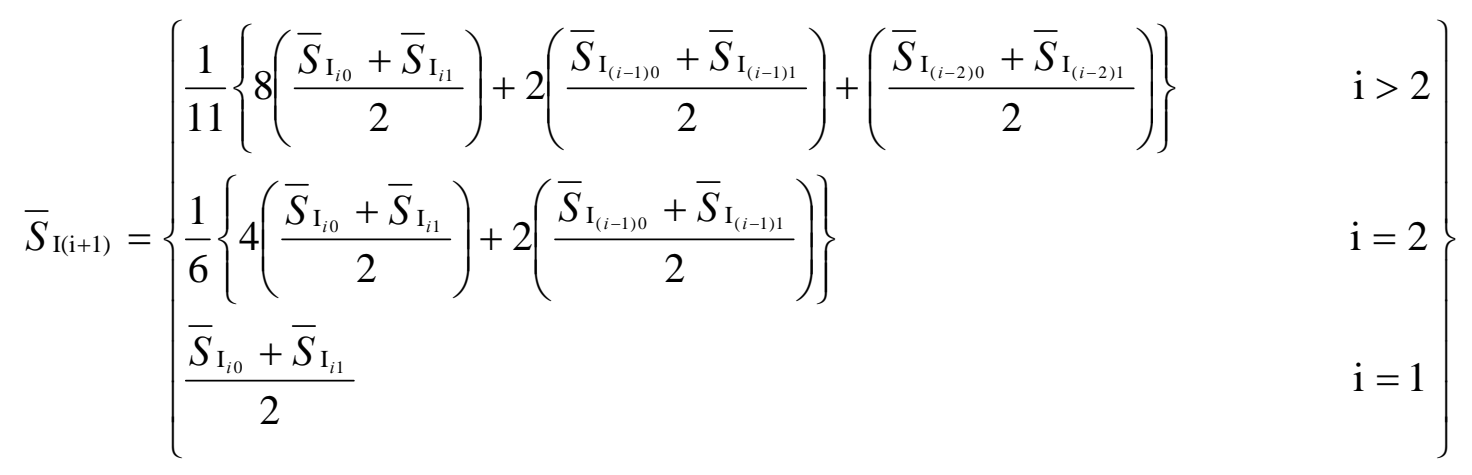

where the terms $\bar{S}_{\mathrm{I}_{\mathrm{j}}}$ are defined in Figure 2 , and $i=1,2, \ldots$ is the number of prior hours of fitted insulin sensitivity values available. The average value $(i=1)$ is obtained using the first two values, $\bar{S}_{\mathrm{I}_{i 0}}$ and $\bar{S}_{\mathrm{I}_{i 1}}$ fitted from 0 to 60 minutes after the first hour of the trial. In subsequent hours, a weighted average of prior fitted values is used to minimise the effect of outliers and/or erroneous glucose measurements on the fitted insulin sensitivity, while emphasising the most current values.

The required combination of bolus size, insulin infusion rate and/or nutritional feed rate to achieve the target glucose level is determined iteratively using the estimated $S_{I}$ 
value, $\bar{S}_{\mathrm{I}_{(i+1)}}$, and Equations (1)-(3). Delta functions are used to model insulin boluses, $u_{e x}(t)$, when solving Equations (1)-(3) with the duration of a bolus set to 1 minute. Similarly, Equation (4) is used to determine $P(t)$ based on the step changes in nutritional input rate.

\section{CLINICAL TRIAL METHODOLOGY}

\subsection{Patient cohort}

All patients were fed enterally only with the mixed-meal formulation RESOURCETM Diabetic (Novartis Medical Nutrition, USA) at a rate not exceeding 700kcal of glucose per day (1944kcal/day total calories) and given infusions of regular human insulin Actrapid ${ }^{\mathrm{TM}}$ (Novo Nordisk Pharmaceuticals Ltd., NZ). Ethical approval was obtained from the Canterbury Ethics Committee.

Selected patients had the following inclusion/exclusion criteria. Inclusion criteria: in situ naso-gastric or other enteral feeding tube (e.g. gastrostomy or naso-jejunal tube); tolerant of the maximal desired feeding rate as determined by the treating clinician; in-patient in intensive care with, or recovering from, a critical illness; random glucose level $>8 \mathrm{mmol} / \mathrm{L}$; age $>16$ years; and an in situ arterial or venous cannula. Exclusion criteria: delayed gastric emptying (high residual 3-hourly gastric aspirates (>200ml); moribund or not expected to survive $>72$ hours; patients receiving neuromuscular blockade (which could theoretically mask agitation or seizures in the event of hypoglycaemia); and morbid obesity $\left(\mathrm{BMI}>35 \mathrm{kgm}^{-2}\right)$. Note that concurrent vasodilator and vasopressor use, and steroid or drug therapy are not exclusion criteria 
for this study as the algorithm developed is intended for broad use across the critical care population. Such conditions modify the insulin sensitivity parameter value as insulin resistance is either elevated or suppressed by the conditions stated and must be adapted to by the algorithm for effective control of hyperglycaemia.

All patients were supine or semi-supine during the trial. Insulin was administered via an intravenous cannula using a Graseby 3500 syringe pump (Graseby Medical Limited, Colonial Way, Watford, Herts, WD24 4LG, UK). Enteral feed rate was maintained and controlled using a Ross Products Patrol Enteral Pump (Abbott Laboratories, Abbott Park, Illinois, U.S.A.).

The proof of concept clinical trial patient cohort $(n=7)$ was selected from the Christchurch ICU intensive care population. This subset represents a heterogeneous cross-section of ICU population, in APACHE and SAP score, age, sex and mortality (see Table 1). The high proportion of septic patients stems from their severity of condition and hence, likelihood of hyperglycaemia. APACHE and SAP scores are calculated from the time at which patient condition is at its worst during the first 24 hours of ICU stay. The mean age is $65.4 \pm 8.1$ years (mean \pm SD), and five out of seven patients are male. These statistics are comparable over this small cohort to other ICUs [2, 55] and an earlier Christchurch ICU study [29]. The ICU survival was obtained subsequently from patient records. 


\subsection{Clinical trial procedure}

The proof-of-concept clinical trials span 10h, from 0900h to $1900 \mathrm{~h}$. The pre-trial period begins at 0600h, at which time the insulin infusion and feed rate are kept constant. The pre-trial period is necessary to obtain an estimate of the patients' onboard insulin level and equilibrium blood glucose, $G_{E}$. At $0900 \mathrm{~h}$, the clinical trial begins and the feed rate is decreased by $30-40 \%$ depending on current glucose level and feed rate. Blood glucose is measured at $15 \mathrm{~min}$ intervals until 1000h. Paired capillary blood samples are taken via finger-prick using lancets and analysed using a bedside Glucocard ${ }^{\mathrm{TM}}$ Test Strip II glucose testing kit (Arkray Inc., Kyoto, Japan). In some cases, arterial blood samples were used when finger pricking was unavailable.

At the end of the first hour, $S_{I}$ is fitted to the first hour of data. Using Equation (6), $S_{I}$ is predicted for the next hour. A target is set for a $10-15 \%$ reduction in current glucose level to a minimum of $5 \mathrm{mmol} / \mathrm{L}$. A combination of insulin bolus size, insulin infusion rate, and enteral feed rate is then resolved to achieve the target at the end of the next hour. Blood glucose is monitored every 30mins, and $S_{I}$ is re-evaluated every hour using the prior hours' data. Following each re-evaluation, the controller determines the required combination of control inputs to achieve the new target depending on insulin resistance and estimated insulin saturation level [18].

Hence, the overall approach is target-driven and incorporates real-time optimisation of insulin sensitivity for adaptive control. The overall clinical trial procedure is outlined in Figure 3. A limit of $6 \mathrm{U}$ is placed on the total insulin prescribed per hour. In addition, a 30mU/L cap was placed on the estimated ineffective insulin, defined by 
the model as the estimated interstitial insulin, $Q$ minus the effective, or saturated insulin, $\bar{Q}$ where $\bar{Q}=\frac{Q}{1+\alpha_{G} Q}$ [18]. Feed rate was limited to between $280 \mathrm{kcal} / \mathrm{day}$ of glucose (778kcal/day total calories) and the newly imposed standard of 700kcal/day of glucose (1944kcal/day total calories) for this ICU. Using the RESOURCETM Diabetic feed formulation, at the $280 \mathrm{kcal} /$ day of glucose minimum, the total caloric intake of $778 \mathrm{kcal} /$ day given a $36 \%$ carbohydrate-based caloric distribution [56] exceeds the caloric intake level found to avoid an increased risk of bloodstream infections [57].

\section{CLINICAL TRIAL RESULTS AND DISCUSSION}

This section presents the results of the proof-of-concept clinical trial tests of the variable feed and insulin control protocol developed.

\subsection{Trial 1}

Patient 1 was a 56-year-old male, Type II diabetic with tetraplegia admitted into intensive care with pneumonia and sepsis. It was his second visit to the ICU and $22^{\text {nd }}$ day of stay. His equilibrium blood glucose level was $12.1 \mathrm{mmol} / \mathrm{L}$ at the start and $11.2 \mathrm{mmol} / \mathrm{L}$ three hours prior. The assumed values of $k, k_{p d}$ and $k_{p r}$ resulted in a smooth fitted $S_{I}$ without further adaptation. The trial progression and modelled patient dynamics are shown in Figure 4 and Table 2.

The insulin distribution volume, $V=12 \mathrm{~L}$, was found to match patient dynamics, and the glucose distribution volume for calculating $P(t)$ was adequately assumed as 
0.19L/kg body mass $(\sim 15 \mathrm{~L})$. The insulin sensitivity remained reasonably constant throughout the first 360mins of the trial, which resulted in hourly-target acquisition with less than $1 \mathrm{mmol} / \mathrm{L}$ absolute error.

Referring to Figure 11, the infusion was varied from 3.0-1.0U/hr and supplemented with three 3.0U boluses. The feed rate was reduced from 700-280kcal/day of glucose (1944-778kcal/day total calories) in the first two hours, which was then maintained until trial completion. After 360mins, the insulin sensitivity began to rise steadily, which affected the accuracy of target acquisition. The last four targets were consistently overestimated, although by not more than $1.0 \mathrm{mmol} / \mathrm{L}$, which was the result of the gradual increase in insulin sensitivity. Note also that the large percentage error is partly due to the much lower than average glucose values.

The gradual increase in identified insulin sensitivity coincided with improved conscious level that may have reflected improved glucose metabolism and increased hepatic glucose uptake, resulting in increased effective insulin sensitivity as seen by the controller. The reduction in blood glucose achieved was $70.2 \%$ from the initial hyperglycaemic $12.1 \mathrm{mmol} / \mathrm{L}$.

\subsection{Trial 2}

Patient 2 was a 64-year-old male, admitted into intensive care post-operatively following cardiac surgery. He has sepsis and acute renal failure. At the time of the trial, it was his $6^{\text {th }}$ day of stay. The trial progression and control inputs are shown in Figure 5 and 11, and Table 2. 
As shown in Figure 11, the feed rate was reduced from the $700-420 \mathrm{kcal} /$ day of glucose (1944-1167kcal/day total calories) in the first hour, but was increased back to $670 \mathrm{kcal} /$ day of glucose (1861kcal/day total calories) at $120 \mathrm{mins}$ to counter the rate of decrease in glucose level. Subsequent feed rate reductions were prescribed at $180 \mathrm{mins}$ to $520 \mathrm{kcal} /$ day of glucose $(1444 \mathrm{kcal} /$ day total calories) and at $240 \mathrm{mins}$ to the $280 \mathrm{kcal} /$ day of glucose minimum (778kcal/day total calories), which was held until trial completion due to the highly insulin resistant nature of the patient, in that period.

Trial 2 highlights some of the difficulties in ICU glycaemic control. From 0 to 120mins, a steady and significant reduction in glucose level was observed stemming from the initial feed rate reduction at 0mins. At 150mins, the glucose measurement was on course for the $180 \mathrm{~min}$ target of $5 \mathrm{mmol} / \mathrm{L}$. However, at $180 \mathrm{mins}$ the measurement was $8.5 \mathrm{mmol} / \mathrm{L}$, which is most likely due to a sudden hyperadrenergic event the patient suffered around that time. The patient suffered a hyperdynamic cardiovascular reaction i.e. atrial fibrillation. This episode highlights how the stress of condition (manifested here in high cardiac output) in the critically ill promotes the counter-regulatory response and sudden changes in glucose level. From that point, the patients' insulin requirement changed with increased effective insulin resistance, requiring much larger insulin boluses after 180mins. Hence, the controller was able to adapt to this significant change in condition and metabolic status that is not atypical in critical care.

Although three targets were missed, the model simulation was tracking the blood glucose measurements accurately within the next hour and the target at 300mins was 
achieved with 5.1\% error. Target acquisition was accurately maintained for the remainder of the trial as the insulin sensitivity remained reasonably constant, albeit relatively low, resulting in a less than $1 \mathrm{mmol} / \mathrm{L}$ absolute target error. Due to the low fitted insulin sensitivity, large feed rate reductions and insulin boluses were required to achieve good control compared to the initial 60-120mins.

The total reduction in blood glucose achieved was $25.3 \%$ from the initial moderately hyperglycaemic $8.7 \mathrm{mmol} / \mathrm{L}$ to a final value of $6.5 \mathrm{mmol} / \mathrm{L}$. The glucose level was irreducible into the 4-6mmol/L target band despite maximising the feed reduction and insulin inputs, emphasising the high insulin resistance encountered through much of the trial period. However, this trial does demonstrate the control algorithms ability to maintain a controlled reduction in glucose level and adapt quickly to rapidly changing patient condition.

Table 2 shows the target errors. The mean error in target acquisition was $15.1 \%$ (range, [0.0, 54.1] \%). Disregarding the large target error at 180mins as patient condition changed suddenly, the average error was $10.2 \%$, which is near the $7-10 \%$ Glucocard $^{\mathrm{TM}}$ measurement errors involved. The average absolute error of $0.68 \mathrm{mmol} / \mathrm{L}$, or $0.92 \mathrm{mmol} / \mathrm{L}$ including the error at $180 \mathrm{mins}$, shows the relatively minor absolute blood glucose errors involved in maintaining tight control.

\subsection{Trial 3}

Patient 3 was a 60-year old male, admitted to the ICU seven days before the trial. He was admitted post-operatively following cardiac surgery with ARDS and multiple 
organ failure, requiring some dialysis. The equilibrium glucose level at the start of the trial was $8.7 \mathrm{mmol} / \mathrm{L}$ and $9.1 \mathrm{mmol} / \mathrm{L}$ one hour beforehand. All parameters were standard values except $k$, which was changed by the controller to $0.0277 \mathrm{~min}^{-1}$ corresponding to a $t_{1 / 2}=25$ mins. This change reduces modelled losses $(n-k)$ thus matching the patients' renal failure condition where the kidneys do not remove as much insulin. The trial progression and control inputs administered are shown in Figure 6 and 11, and Table 2.

The control algorithm prescribed a feed rate cut from 545-318kcal/day of glucose (1514-883kcal/day total calories) in the first 120 mins but eventually increased the feed rate back to $545 \mathrm{kcal} /$ day of glucose (1514kcal/day total calories) at $240 \mathrm{mins}$ (range, [273, 545] kcal/day of glucose, [758, 1514] kcal/day total calories). Effective fitted insulin sensitivity was sufficiently high that insulin therapy was adequate for the desired glucose reduction and large feed rate reductions were unnecessary. Insulin boluses administered ranged from 1.5-4.0U. Insulin infusion was reduced from 21U/hr at 360mins and maintained until trial completion. The reduction in blood glucose achieved was $35.8 \%$ from the initial value of $8.7 \mathrm{mmol} / \mathrm{L}$ to the final value of $5.6 \mathrm{mmol} / \mathrm{L}$. The mean error of target acquisition is $7.2 \%$ (range: $0.7-21.9 \%$ ) and the maximum absolute error recorded was $0.86 \mathrm{mmol} / \mathrm{L}$.

Patient 3 exhibited higher effective insulin sensitivity than both Patient 1 and 2, which is confirmed by the smaller sized and lower frequency of insulin boluses administered in the trial, as well as the feed rate modulation required for the desired glucose level reduction. Equally importantly, this trial demonstrated the controllers' ability to capture the renal failure condition and reduced insulin losses. It also was able to 
determine that insulin alone would achieve tight control, thus not interrupting nutritional input.

\subsection{Trial 4}

Patient 4 was a 75-year old non-diabetic female with renal and multiple organ failure from sepsis, and required some dialysis. She had spent 23 days in ICU. The equilibrium glucose level at the start of the trial period was a normal $4.8 \mathrm{mmol} / \mathrm{L}$, although she had mild hyperglycaemia with a blood glucose level of $7.2 \mathrm{mmol} / \mathrm{L}$ two hours earlier. With normal initial blood glucose, the controller would be trialled in maintaining tight control throughout the 10-hour trial period, which would not be possible if the initial blood glucose level was hyperglycaemic. The results are shown in Figure 7 and 11, and Table 2.

The resulting fitted insulin sensitivity was smooth with only minor discontinuities between each fit. The insulin infusion was reduced from 2.0-1.0U/hr at 360mins into the trial. One insulin bolus was administered at 480mins, $1.0 \mathrm{U}$ in size. The feed rate was reduced from the initial 455kcal/day of glucose (1264kcal/day total calories) and modulated (range, [364, 636] kcal/day of glucose, [1011, 1767] kcal/day total calories), with the same final as initial feed rate.

For the first 5 hours, the glucose level was maintained in the desired 4-6mmol/L band. However, a sudden shift in patient condition occurred at 300mins. Note that up until that point, no insulin boluses had been administered, the insulin infusion was a constant $2 \mathrm{U} / \mathrm{hr}$ since the start of the trial, and only small feed rate changes had been 
performed. A drop in glucose level occurred to $3.4 \mathrm{mmol} / \mathrm{L}$ (a target error of $33 \%$ or $1.7 \mathrm{mmol} / \mathrm{L}$ ). An increase in feed rate to $636 \mathrm{kcal} /$ day and a reduction in the insulin infusion to $1 \mathrm{U} / \mathrm{hr}$ were then prescribed by the control algorithm. The drop in glucose level was halted at 360mins and glucose level remained within the 4-6mmol/L desired band until the trial end. The cause of the sudden increase in insulin sensitivity at 300mins is unclear, but may be related to reduced insulin clearance by the kidneys. However, as in Trial 2, the model adapted within 1 hour, with the final two measurements having a mean error of $6.8 \%(0.3 \mathrm{mmol} / \mathrm{L}$ absolute).

The reduction in blood glucose achieved was $30.1 \%$ from the initial mild hyperglycaemia of $6.8 \mathrm{mmol} / \mathrm{L}$ to a final value of $4.75 \mathrm{mmol} / \mathrm{L}$. With significantly higher insulin sensitivity compared to Patient 2, the glucose level was controlled in the 4-6mmol/L target band, largely with insulin, resorting to a feed rate increase only when fitted insulin sensitivity increased significantly.

The mean target error was 9.9\% (range, [0.2, 33.0] \%) with a maximum absolute error of $1.65 \mathrm{mmol} / \mathrm{L}$. Neglecting the few errors arising from the rapid increase in insulin sensitivity, the mean error reduces to $5.0 \%$. The fitted insulin sensitivity distribution remained relatively constant, which contributed to the high rate of successful target acquisition. Overall, the errors were very low and all targets were met.

\subsection{Trial 5}

Patient 5 was a 73-year old, insulin dependent Type II diabetic male admitted into ICU with aspiration pneumonia secondary to mediastinal sepsis and an 
oesophagectomy. The patient had spent 29 days in ICU, and the equilibrium glucose level at the start of the trial period was $6.8 \mathrm{mmol} / \mathrm{L}$, although he had significantly elevated blood glucose levels $(9-10 \mathrm{mmol} / \mathrm{L})$ earlier. With near normal initial blood glucose, the controller would again be trialled in maintaining tight control throughout the 10-hour trial period. The results are shown in Figure 8 and 11, and Table 2.

The resulting fitted insulin sensitivity was very smooth with only minor discontinuities between each fit. The insulin infusion was kept constant throughout the trial at the initial value of $1.5 \mathrm{U} / \mathrm{hr}$, except for a one hour period, and supplemented with six boluses of $0.5-1.5 \mathrm{U}$. The feed rate was reduced from $550-365 \mathrm{kcal} / \mathrm{day}$ of glucose (1528-1014kcal/day total calories) for the first four hours and increased back to $464 \mathrm{kcal} /$ day of glucose (1289kcal/day total calories) by the end of the trial. For the first 5 hours, the blood glucose was reduced and then held at $5 \mathrm{mmol} / \mathrm{L}$.

The rise in blood glucose between 300mins and 480mins was caused by a miscommunication. The control input at 300mins as determined by the controller was to maintain the insulin infusion at $1.5 \mathrm{U} / \mathrm{hr}$ and administer a $0.6 \mathrm{U}$ insulin bolus, but was misconstrued by the attending nurse as reduce the insulin infusion to $0.6 \mathrm{U} / \mathrm{hr}$ only. The resultant increase in blood glucose was halted at 420mins into the trial when the controller was given the correct values. Even so, the controller predicted the rise in glucose accurately when the data was updated.

The reduction in blood glucose achieved was $30.1 \%$ from the initial mild hyperglycaemia of $6.8 \mathrm{mmol} / \mathrm{L}$ to a final value of $4.75 \mathrm{mmol} / \mathrm{L}$. The mean target error was $2.3 \%$ (range: $0-5 \%$ ) and a maximum absolute error of $0.25 \mathrm{mmol} / \mathrm{L}$ was recorded. 
The insulin sensitivity distribution for the whole trial remained relatively constant, which contributed to the high rate of successful target acquisition. Overall, the errors were very low and all targets were met.

\subsection{Trial 6}

Patient 6 was a 57-year old male admitted into the ICU with pneumococcal meningitis. He also suffered a stroke, arising from his inflammatory condition. He had spent 6 days in ICU. His equilibrium glucose level at the start of the trial period was $7.5 \mathrm{mmol} / \mathrm{L}$, and $7.0 \mathrm{mmol} / \mathrm{L}$ at the start of the 3-hour pre-trial period. The results are shown in Figure 9 and 11, and Table 2.

The resulting fitted insulin sensitivity was very smooth with only minor discontinuities between each fit. At 120mins, the enteral feed was disconnected in anticipation of the patient being transferred for an immediate MRI scan as requested by the attending physician. The insulin infusion was unchanged at the initial $2 \mathrm{U} / \mathrm{hr}$. At 180mins, the trial was interrupted and the insulin infusion was disconnected. At 318mins, the patient was returned and the insulin infusion and enteral feed restarted at a rate of $0.5 \mathrm{U} / \mathrm{hr}$ and $280 \mathrm{kcal} /$ day, respectively. These values were maintained until the end of the trial.

One unpaired glucose measurement was obtained at 270mins while the patient was undergoing the MRI scan. From the model simulation, it is likely that this measurement was inaccurate given that the target at 300mins was within the model simulation. Before the scan, two insulin boluses of 1.1 and $2.0 \mathrm{U}$ with a constant 
$2 \mathrm{U} / \mathrm{hr}$ infusion achieved a reduction in glucose level to $5.7 \mathrm{mmol} / \mathrm{L}$ with the initial 827-545kcal/day of glucose (2297-1514kcal/day total calories) feed rate cut at 0mins. After the scan, the patient became noticeably more insulin resistant, as shown by the insulin sensitivity plot. The feed rate was reduced to its minimum level of $280 \mathrm{kcal} /$ day of glucose (778kcal/day total calories) and bolus size was increased up to 5.5U. In the last five hours of the trial, the average insulin administered was 5.0U/hr compared to $1.8 \mathrm{U} / \mathrm{hr}$ in the first five hours.

The reduction in blood glucose achieved was $30.7 \%$ from the initial mild hyperglycaemia of $7.5 \mathrm{mmol} / \mathrm{L}$ to a final value of $5.2 \mathrm{mmol} / \mathrm{L}$. With moderate insulin sensitivity, the glucose level was reduced and maintained in the $4-6 \mathrm{mmol} / \mathrm{L}$ target band with a combination of insulin and feed rate reduction.

The mean target error was $7.6 \%$ (range, $[1.3,15.0] \%$ ) with a maximum absolute error of $0.75 \mathrm{mmol} / \mathrm{L}$. The reduction in insulin sensitivity observed after the MRI scan may have resulted from transient increases in carbon dioxide when the patient was ventilated using a basic transport ventilator. However, the insulin sensitivity distribution for the whole trial remained relatively constant, which contributed to the high rate of successful target acquisition.

\subsection{Trial 7}

Patient 7 was a 73-year old female admitted into the ICU with an incarcerated hernia, acute renal failure and metabolic acidosis. The patient had spent 3 days in ICU, and the equilibrium glucose level at the start of the trial period was $9.9 \mathrm{mmol} / \mathrm{L}$, and 
$10.3 \mathrm{mmol} / \mathrm{L}$ at the start of the pre-trial period. The results are shown in Figure 10 and 11, and Table 2.

The resulting fitted insulin sensitivity was very smooth with only minor discontinuities between each fit. Initially, the effective insulin sensitivity was low, but at 240mins it began to gradually increase, reducing the average total insulin required per hour. The enteral feed rate was reduced from the initial 545kcal/day of to $318 \mathrm{kcal} /$ day of glucose at 0 mins (1514-883kcal/day total calories) and was maintained until the end of the trial.

As a result of the increase in fitted insulin sensitivity, at 420mins the $5 \mathrm{mmol} / \mathrm{L}$ target glucose level was overestimated by $0.95 \mathrm{mmol} / \mathrm{L}(4.05 \mathrm{mmol} / \mathrm{L}$ measured $)$, resulting in the insulin infusion being reduced to $0.5 \mathrm{U} / \mathrm{hr}$ from the initial constant $3.0 \mathrm{U} / \mathrm{hr}$. The feed rate was kept at 318kcal/day. A 3.0U bolus was then administered at 480mins and the patients' blood glucose was kept in the $4-6 \mathrm{mmol} / \mathrm{L}$ band until the end of the trial.

The mean target error was $7.5 \%$ (range, $[1.0,19.0] \%$ ) and a maximum absolute error of $0.95 \mathrm{mmol} / \mathrm{L}$ was recorded. The reduction in blood glucose achieved was $49.0 \%$ from the initial $9.9 \mathrm{mmol} / \mathrm{L}$ to a final value of $5.1 \mathrm{mmol} / \mathrm{L}$. With moderate insulin sensitivity, the glucose level was reduced and maintained in the $4-6 \mathrm{mmol} / \mathrm{L}$ target band with a combination of insulin and feed rate reduction. 


\subsection{Control method and target acquisition error analysis}

The overall mean target error for all trials was $8.9 \%(0.51 \mathrm{mmol} / \mathrm{L})$, with an absolute range of $[0,2.9] \mathrm{mmol} / \mathrm{L}$. The Glucocard ${ }^{\mathrm{TM}}$ Test Strip II bedside glucose monitoring sensor is capable of obtaining $50 \%$ and $98 \%$ of measurements within $\pm 5 \%$ and $\pm 20 \%$ error respectively, over typical glucose ranges [58]. Across the 7 clinical trials, 43\% of the targets were achieved within $\pm 5 \%$ with a mean target error of $2.3 \%$ $(0.15 \mathrm{mmol} / \mathrm{L})$. Mean target error for errors $>5 \%$ was $13.8 \%(0.79 \mathrm{mmol} / \mathrm{L})$. Out of the 63 targets, only six had errors larger than $20 \%$, so that $91 \%$ of all measurements were within $\pm 20 \%$ of targets. However, all of these errors can be explained by significant, rapid changes in patient condition, rather than pure measurement errors. The target errors obtained are thus consistent with, and explainable, by published measurement errors from literature.

Figure 12 presents a bootstrapped linear regression model, as applied to the achieved and target glucose values. The estimated standard error of the regression model, $s$, is the test statistic shown in Equation (7).

$s=\frac{\sum_{i=1}^{n}\left(y_{i}-\hat{y}_{i}\right)^{2}}{n-2}$

where $n=63$ and is the bootstrap sample size ( 9 targets for each of the 7 trials), $y_{i}$ is the achieved glucose value recorded for the $i$ th target glucose value, $x_{i}$, and $\hat{y}_{i}$ is the value of the least squares linear regression curve at the target glucose value, $x_{i}$. 
The number of bootstrap samples was 4000 . Figure 12 also shows the $95 \%$ confidence limits for the prediction of the achieved glucose values for a given target value. The few glucose data points achieved with high accuracy at glucose concentrations $>7 \mathrm{mmol} / \mathrm{L}$ resulted in a very small effective variation in the bootstrap estimate. Conversely, the number of outliers from rapid changes in patient condition from the greater number of data points around normal glucose levels resulted in a larger variation in the bootstrap estimate. This result explains the confidence bands, which are nearly of equal width across the glucose range shown.

Note that this analysis is necessarily conservative, as outliers from rapid changes in patient condition were included. The non-parametric bootstrap confidence intervals point to a slight bias towards overshooting the target glucose concentration, which may be deemed safer in a clinical environment. However, this result cannot be confirmed with the current sample size $(n=63)$. A white residual plot based on the linear regression curve showed no systematic error in the model and methods as shown in Figure 13. A correlation coefficient, $R$, between 0.7820 and 0.9216 can be stated with 95\% confidence. A larger data point sample size from ongoing trials will confirm whether current target errors are within reported sensor parameters or partly due to other systematic problems.

Finally, it is also important to note the affect of patient cohort, and in particular level of illness as measured by APACHE II score, on the results. Overall, the clinical results showed tight control to less than $6.1 \mathrm{mmol} / \mathrm{L}$ for a cohort with median APACHE II score of 23 (range, [17, 31]). In comparison, van den Berghe et al [2] achieved similarly tight control with median APACHE II score of 9 (inter-quartile 
range, [7, 13]), which represents a much lower level of illness. For a more comparable ICU population, Krinsley [13, 14] showed tight control to a higher 7.75mmol/L for a cohort with median APACHE II of 16 (inter-quartile range, [10, 23]). However, both these studies used insulin alone to control glucose levels. Hence, the significantly added control obtained by modulating nutrition, as well as insulin, to control glycaemic levels is seen in the ability to achieve tight control to a level similar to [2] for a more critically ill ICU cohort.

\section{CONCLUSIONS}

The clinical pilot trials conducted during this study demonstrated the potential of a control algorithm modulating both insulin and nutritional feed rate to accurately reduce and tightly regulate glucose levels despite significant inter-patient variability and time-varying physiological condition. A simple, two-compartment model for the enteral feed rate was presented and used to simply model variations in nutritional input.

The efficacy of the adaptive control algorithm and system model presented in achieving targeted control of hyperglycaemia in critically ill patients was validated in seven clinical, proof-of-concept pilot trials. The results showed both accurate regulated stepwise glycaemic reduction and tight glycaemic maintenance with a mean target error of $8.9 \%$. All target errors above $20 \%$ were attributable to periods where the controller was adapting to sudden changes in patient physiology, rather than systematic deficiencies in the model or protocol while all target errors were consistent with and explainable by the published sensor error distributions. Notably, the average 
absolute error was $0.51 \mathrm{mmol} / \mathrm{L}$, a small value compared to the $4-6 \mathrm{mmol} / \mathrm{L}$ desired, especially in a clinical environment where target error of $15-20 \%$ is not considered clinically significant.

A larger patient cohort needs to be tested with the current model and control algorithm to further analyse its effectiveness and improve its performance. Trials spanning longer periods of time are also in development to verify the long-term performance of the control algorithm and protocol developed, and to further test the adaptability of the controller. Clinically, these results have the potential to reduce ICU mortality and the risk of severe long-term complications with relatively limited clinical effort.

\section{ACKNOWLEDGEMENTS}

This research was funded by a Tertiary Education Commission (TEC) Top Achiever

Doctoral Scholarship and the Canterbury Intensive Care Research and Education Trust.

\section{REFERENCES}

[1] S. E. Capes, D. Hunt, K. Malmberg, and H. C. Gerstein, "Stress hyperglycaemia and increased risk of death after myocardial infarction in patients with and without diabetes: a systematic overview," Lancet, vol. 355, pp. 773-778, 2000.

[2] G. Van den Berghe, P. Wouters, F. Weekers, C. Verwaest, F. Bruyninckx, M. Schetz, D. Vlasselaers, P. Ferdinande, P. Lauwers, and R. Bouillon, "Intensive insulin therapy in the critically ill patients," N Engl J Med, vol. 345, pp. 13591367, 2001. 
[3] B. A. Mizock, "Alterations in fuel metabolism in critical illness: hyperglycaemia," Best Pract Res Clin Endocrinol Metab, vol. 15, pp. 533-51, 2001.

[4] K. C. McCowen, A. Malhotra, and B. R. Bistrian, "Stress-induced hyperglycemia," Crit Care Clin, vol. 17, pp. 107-24, 2001.

[5] J. F. Patino, S. E. de Pimiento, A. Vergara, P. Savino, M. Rodriguez, and J. Escallon, "Hypocaloric support in the critically ill," World J Surg, vol. 23, pp. 553-9, 1999.

[6] C. Weissman, "Nutrition in the intensive care unit," Crit Care, vol. 3, pp. R6775, 1999.

[7] A. M. Woolfson, "Control of blood glucose during nutritional support in ill patients," Intensive Care Med, vol. 7, pp. 11-4, 1980.

[8] M. Elia, A. Ceriello, H. Laube, A. J. Sinclair, M. Engfer, and R. J. Stratton, "Enteral Nutritional Support and Use of Diabetes-Specific Formulas for Patients With Diabetes: A systematic review and meta-analysis," Diabetes Care, vol. 28, pp. 2267-79, 2005.

[9] B. J. Krajicek, Y. C. Kudva, and H. A. Hurley, "Potentially important contribution of dextrose used as diluent to hyperglycemia in hospitalized patients," Diabetes Care, vol. 28, pp. 981-2, 2005.

[10] A. Thorburn, A. Litchfield, S. Fabris, and J. Proietto, "Abnormal Transient Rise in Hepatic Glucose-Production after Oral Glucose in Non-InsulinDependent Diabetic Subjects," Diabetes Research and Clinical Practice, vol. 28, pp. 127-135, 1995.

[11] M. O. Larsen, B. Rolin, M. Wilken, R. D. Carr, and O. Svendsen, "High-fat high-energy feeding impairs fasting glucose and increases fasting insulin levels in the Gottingen minipig - Results from a pilot study," in Lipids and Insulin Resistance: The Role of Fatty Acid Metabolism and Fuel Partitioning, vol. 967, Annals of the New York Academy of Sciences, 2002, pp. 414-423.

[12] B. R. Bistrian, "Hyperglycemia and Infection: Which is the Chicken and Which is the Egg?," JPEN J Parenter Enteral Nutr, vol. 25, pp. 180-181, 2001.

[13] M. N. Diringer, "Improved outcome with aggressive treatment of hyperglycemia - Hype or hope?," Neurology, vol. 64, pp. 1330-1331, 2005.

[14] G. Van den Berghe, P. J. Wouters, R. Bouillon, F. Weekers, C. Verwaest, M. Schetz, D. Vlasselaers, P. Ferdinande, and P. Lauwers, "Outcome benefit of intensive insulin therapy in the critically ill: Insulin dose versus glycemic control," Crit Care Med, vol. 31, pp. 359-366, 2003.

[15] J. S. Krinsley, "Decreased mortality of critically ill patients with the use of an intensive glycemic management protocol," Crit Care Med, vol. 31, pp. A19, 2003. 
[16] J. S. Krinsley, "Effect of an intensive glucose management protocol on the mortality of critically ill adult patients," Mayo Clin Proc, vol. 79, pp. 9921000, 2004.

[17] C. E. Hann, J. G. Chase, J. Lin, T. Lotz, C. V. Doran, and G. M. Shaw, "Integral-based parameter identification for long-term dynamic verification of a glucose-insulin system model," Comput Methods Programs Biomed, vol. 77, pp. 259-70, 2005.

[18] J. G. Chase, G. M. Shaw, J. Lin, C. V. Doran, C. Hann, M. B. Robertson, P. M. Browne, T. Lotz, G. C. Wake, and B. Broughton, "Adaptive bolus-based targeted glucose regulation of hyperglycaemia in critical care," Med Eng Phys, vol. 27, pp. 1-11, 2005.

[19] S. Rashid, Z. Q. Shi, M. Niwa, J. M. R. Mathoo, M. Vandelangeryt, D. Bilinski, G. F. Lewis, and M. Vranic, "beta-blockade, but not normoglycemia or hyperinsulinemia, markedly diminishes stress-induced hyperglycemia in diabetic dogs," Diabetes, vol. 49, pp. 253-262, 2000.

[20] S. Rashid, M. Niwa, M. VanDelangeryt, J. Mathoo, D. Bilinski, and M. Vranic, "Improvement of impaired glucose clearance during stress in diabetes with hyperinsulinemia or beta-blockade," Diabetes, vol. 46, pp. 881-881, 1997.

[21] R. L. Prigeon, M. E. Roder, D. Porte, Jr., and S. E. Kahn, "The effect of insulin dose on the measurement of insulin sensitivity by the minimal model technique. Evidence for saturable insulin transport in humans," J Clin Invest, vol. 97, pp. 501-507, 1996.

[22] A. Natali, A. Gastaldelli, S. Camastra, A. M. Sironi, E. Toschi, A. Masoni, E. Ferrannini, and A. Mari, "Dose-response characteristics of insulin action on glucose metabolism: a non-steady-state approach," Am J Physiol Endocrinol Metab, vol. 278, pp. E794-801, 2000.

[23] A. Mari and A. Valerio, "A Circulatory Model for the Estimation of Insulin Sensitivity," Control Eng Practice, vol. 5, pp. 1747-1752, 1997.

[24] J. C. Beard, R. N. Bergman, W. K. Ward, and D. J. Porte, "The insulin sensitivity index in nondiabetic man. Correlation between clamp-derived and IVGTT-derived values," Diabetes, vol. 35, pp. 362-369, 1986.

[25] A. Caumo, P. Vicini, J. J. Zachwieja, A. Avogaro, K. Yarasheski, D. M. Bier, and C. Cobelli, "Undermodeling affects minimal model indexes: insights from a two-compartment model," Am J Physiol, vol. 276, pp. E1171-1193, 1999.

[26] R. Hovorka, V. Canonico, L. J. Chassin, U. Haueter, M. Massi-Benedetti, M. O. Federici, T. R. Pieber, H. C. Schaller, L. Schaupp, T. Vering, and M. E. Wilinska, "Nonlinear model predictive control of glucose concentration in subjects with type 1 diabetes," Physiological Measurement, vol. 25, pp. 905920, 2004. 
[27] F. Chee, T. Fernando, and P. V. van Heerden, "Closed-loop control of blood glucose levels in critically ill patients," Anaesth Intensive Care, vol. 30, pp. 295-307, 2002.

[28] C. V. Doran, N. H. Hudson, K. T. Moorhead, J. G. Chase, G. M. Shaw, and C. E. Hann, "Derivative weighted active insulin control modelling and clinical trials for ICU patients," Medical Engineering \& Physics, vol. 26, pp. 855-866, 2004.

[29] C. V. Doran, "Modelling and Control of Hyperglycemia in Critical Care Patients," in Mechanical Engineering. Christchurch, NZ: University of Canterbury, 2004.

[30] E. Kiwanuka, R. Barazzoni, and P. Tessari, "Glucose kinetics and splanchnic uptake following mixed meal ingestion in cirrhotic-diabetic subjects," Diabetes Nutrition \& Metabolism, vol. 14, pp. 315-324, 2001.

[31] R. R. Wolfe, "Radioactive and stable isotope tracers in biomedicine," Principles and practice of kinetic analysis, pp. 119-144, 1992.

[32] R. A. DeFronzo, J. D. Tobin, and R. Andres, "Glucose clamp technique: a method for quantifying insulin secretion and resistance," Am J Physiol, vol. 237, pp. E214-23, 1979.

[33] B. Thorsteinsson, S. Fugleberg, B. Feldt-Rasmussen, K. Ellemann, and C. Binder, "Kinetics Models for Insulin Disappearance from Plasma in Type 1 Diabetic Patients," Pharmacol Toxicol, vol. 60, pp. 90-95, 1987.

[34] R. N. Bergman, L. S. Phillips, and C. Cobelli, "Physiologic evaluation of factors controlling glucose tolerance in man: measurement of insulin sensitivity and beta-cell glucose sensitivity from the response to intravenous glucose," J Clin Invest, vol. 68, pp. 1456-1467, 1981.

[35] K. Ellemann, B. Thorsteinsson, S. Fugleberg, B. Feldt-Rasmussen, O. O. Andersen, P. Gronbaek, and C. Binder, "Kinetics of insulin disappearance from plasma in cortisone-treated normal subjects," Clin Endocrinol (Oxf), vol. 26, pp. 623-628, 1987.

[36] L. Kuehn and T. Blundell, "Insulin and glucagons receptors," in Cellular receptors for hormones and neurotransmitters, D. Schulster and A. Levitski, Eds. Chichester: John Wiley and Sons, 1980, pp. 185-196.

[37] R. A. Rizza, L. J. Mandarino, and J. E. Gerich, "Dose-response characteristics for effects of insulin on production and utilization of glucose in man," Am J Physiol, vol. 240, pp. E630-639, 1981.

[38] R. Hovorka, F. Shojaee-Moradie, P. V. Carroll, L. J. Chassin, I. J. Gowrie, N. C. Jackson, R. S. Tudor, A. M. Umpleby, and R. H. Jones, "Partitioning glucose distribution/transport, disposal, and endogenous production during IVGTT," Am J Physiol Endocrinol Metab, vol. 282, pp. E992-1007, 2002. 
[39] R. N. Bergman, D. T. Finegood, and M. Ader, "Assessment of insulin sensitivity in vivo," Endocr Rev, vol. 6, pp. 45-86, 1985.

[40] S. M. Furler, E. W. Kraegen, R. H. Smallwood, and D. J. Chisholm, "Blood glucose control by intermittent loop closure in the basal mode: computer simulation studies with a diabetic model," Diabetes Care, vol. 8, pp. 553-561, 1985.

[41] K. Transberg, P. Hagander, and J. Thorell, "Disappearance of insulin in man: variation with the plasma insulin level," Acta Endocrinol (Copenh), vol. 97, pp. 391-397, 1981.

[42] B. Ludvik, J. J. Nolan, A. Roberts, J. Baloga, M. Joyce, J. M. Bell, and J. M. Olefsky, "Evidence for decreased splanchnic glucose uptake after oral glucose administration in non-insulin-dependent diabetes mellitus," Journal of Clinical Investigation, vol. 100, pp. 2354-2361, 1997.

[43] J. Radziuk, T. J. McDonald, D. Rubenstein, and J. Dupre, "Initial Splanchnic Extraction of Ingested Glucose in Normal Man," Metabolism-Clinical and Experimental, vol. 27, pp. 657-669, 1978.

[44] B. Ludvik, J. J. Nolan, A. Roberts, J. Baloga, M. Joyce, J. M. Bell, and J. M. Olefsky, "A Noninvasive Method to Measure Splanchnic Glucose-Uptake after Oral Glucose-Administration," Journal of Clinical Investigation, vol. 95, pp. 2232-2238, 1995.

[45] A. J. Cummins, "Absorption of glucose and methionine from the human intestine. Influence of the glucose concentration in the blood and the intestinal lumen," J. Clin. Invest., vol. 31, pp. 928-937, 1952.

[46] A. Consoli, "Role of Liver in Pathophysiology of Niddm," Diabetes Care, vol. 15, pp. 430-441, 1992.

[47] G. Pehling, P. Tessari, J. E. Gerich, M. W. Haymond, F. J. Service, and R. A. Rizza, "Abnormal Meal Carbohydrate Disposition in Insulin-Dependent Diabetes - Relative Contributions of Endogenous Glucose-Production and Initial Splanchnic Uptake and Effect of Intensive Insulin Therapy," Journal of Clinical Investigation, vol. 74, pp. 985-991, 1984.

[48] A. Mari, L. Stojanovska, J. Proietto, and A. W. Thorburn, "A circulatory model for calculating non-steady-state glucose fluxes. Validation and comparison with compartmental models," Computer Methods and Programs in Biomedicine, vol. 71, pp. 269-281, 2003.

[49] R. Steele, "Influence of glucose loading and of injected insulin on hepatic glucose output," Ann. N. Y. Acad. Sci., vol. 82, pp. 420-430, 1959.

[50] M. F. Nielsen, R. Basu, S. Wise, A. Caumo, C. Cobelli, and R. A. Rizza, "Normal glucose-induced suppression of glucose production but impaired stimulation of glucose disposal in type 2 diabetes: evidence for a concentration-dependent defect in uptake," Diabetes, vol. 47, pp. 1735-47, 1998. 
[51] R. Basu, A. Basu, C. M. Johnson, W. F. Schwenk, and R. A. Rizza, "Insulin dose-response curves for stimulation of splanchnic glucose uptake and suppression of endogenous glucose production differ in nondiabetic humans and are abnormal in people with type 2 diabetes," Diabetes, vol. 53, pp. 20422050, 2004.

[52] A. Basu, R. Basu, P. Shah, A. Vella, C. M. Johnson, M. Jensen, K. S. Nair, W. F. Schwenk, and R. A. Rizza, "Type 2 diabetes impairs splanchnic uptake of glucose but does not alter intestinal glucose absorption during enteral glucose feeding - Additional evidence for a defect in hepatic glucokinase activity," Diabetes, vol. 50, pp. 1351-1362, 2001.

[53] R. Firth, P. Bell, H. Marsh, I. Hansel, and R. Rizza, "Post-prandial hyperglycemia in patients with non-insulin dependent diabetes mellitus: role of hepatic and extrahepatic tissues," J. Clin. Invest., vol. 74, pp. 1525-1532, 1985.

[54] T. Arleth, S. Andreassen, M. Orsini Federici, A. Timi, and M. MassiBenedetti, "A model of glucose absorption from mixed meals," Modelling and Control of Biomedical Systems 2000 (including Biological Systems): A Proceedings Volume from the 4th IFAC Symposium, 2000, E. Carson and E. Salzsieder, Elsevier Science Limited, Oxford, pp. 307-312.

[55] J. S. Krinsley, "Association between hyperglycemia and increased hospital mortality in a heterogeneous population of critically ill patients," Mayo Clin Proc, vol. 78, pp. 1471-1478, 2003.

[56] Novartis, "RESOURCE® Diabetic Nutrition Information." US: Novartis Medical Nutrition, 2005.

[57] L. Rubinson, G. B. Diette, X. Song, R. G. Brower, and J. A. Krishnan, "Low caloric intake is associated with nosocomial bloodstream infections in patients in the medical intensive care unit," Crit Care Med, vol. 32, pp. 350-7, 2004.

[58] R. Weitgasser, B. Gappmayer, and M. Pichler, "Newer portable glucose meters - Analytical improvement compared with previous generation devices?," Clinical Chemistry, vol. 45, pp. 1821-1825, 1999. 


\section{FIGURES}

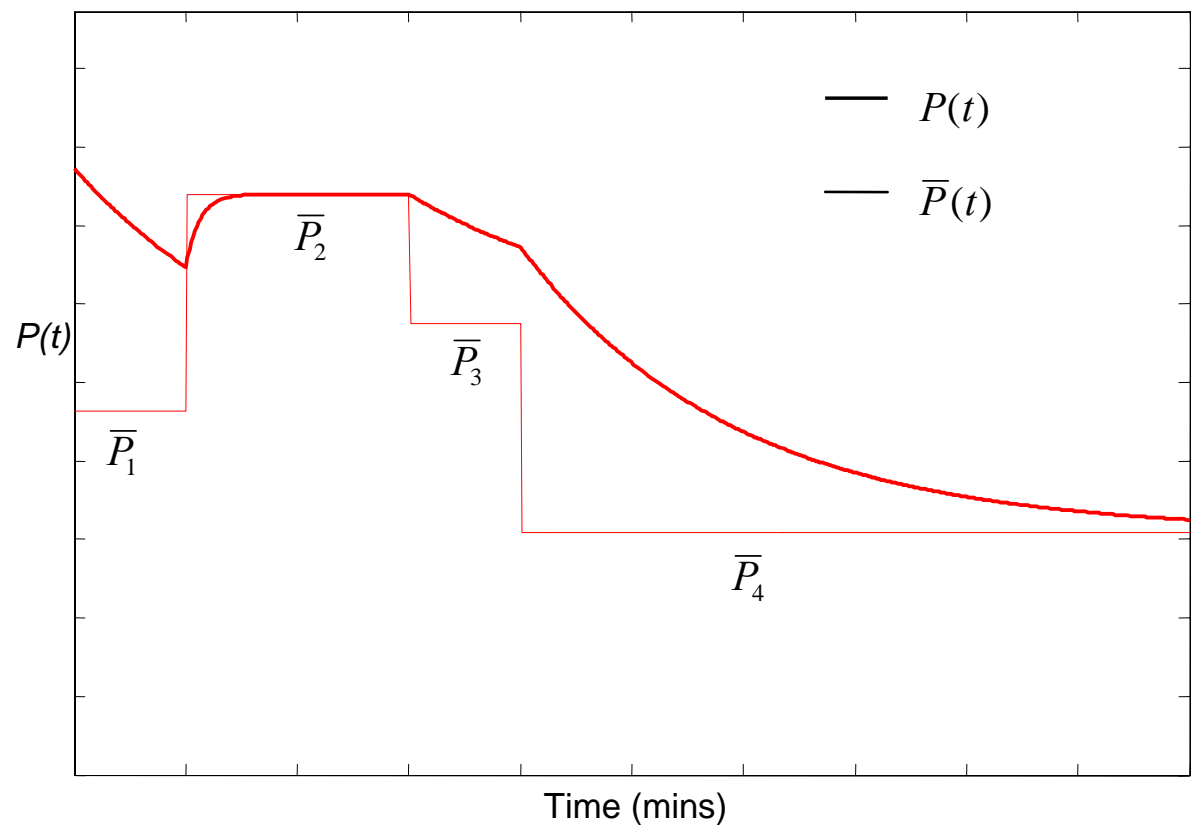

Figure 1: Stepwise enteral nutritional variation and modelled total plasma nutritional input, $P(t)$ 


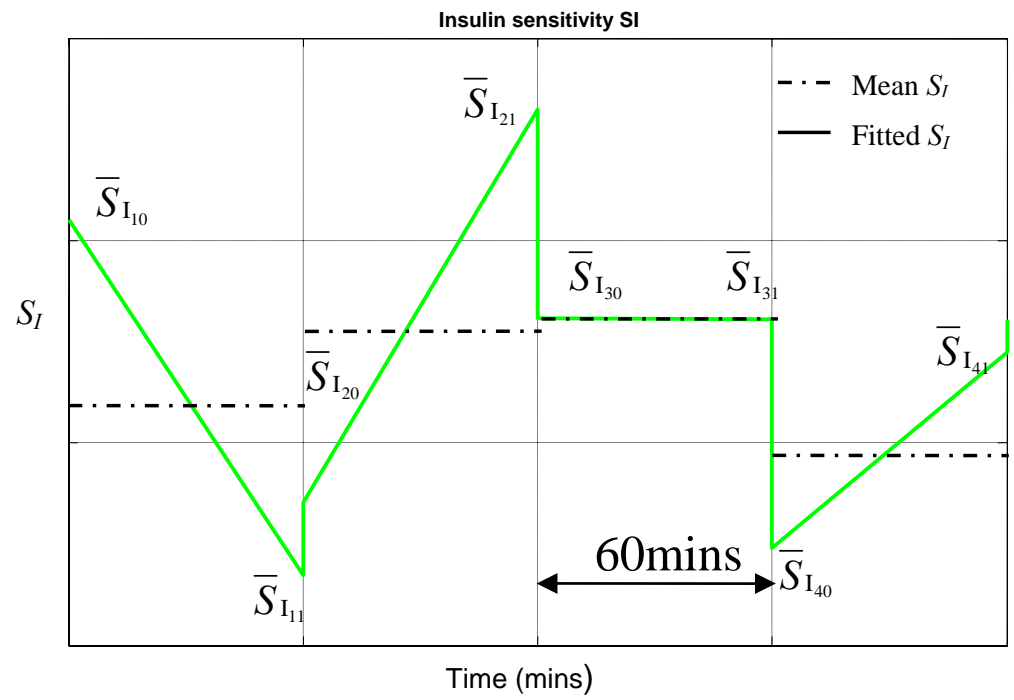

Figure 2: Example of fitted insulin sensitivity value definitions, right most values are most recent, going back 4 hours. Values are calculated using Equation (6) 


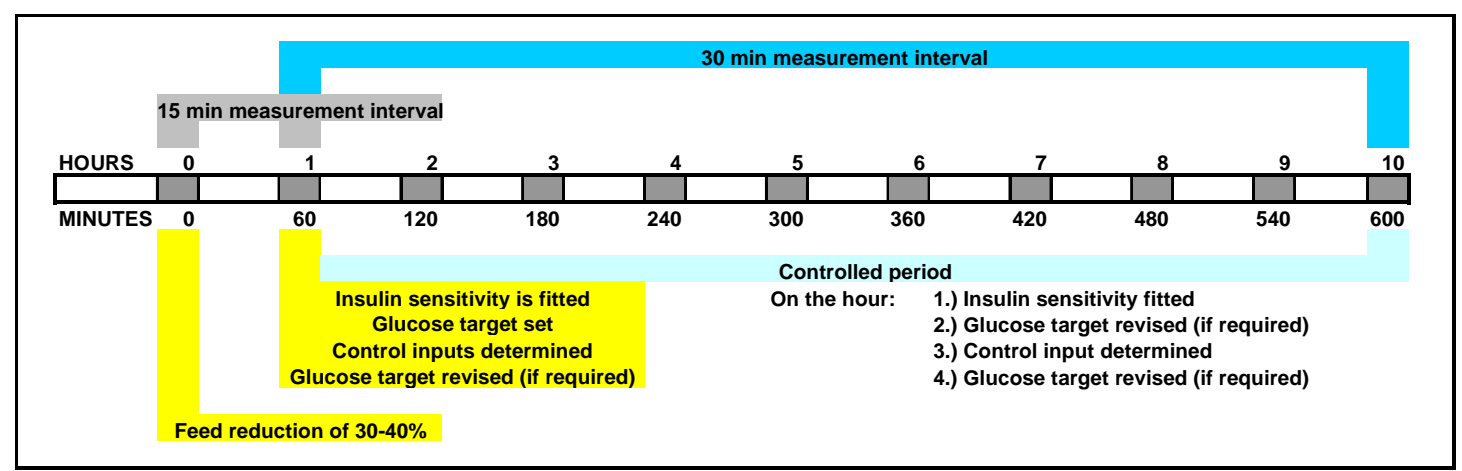

Figure 3: 10-hour clinical trial procedure 


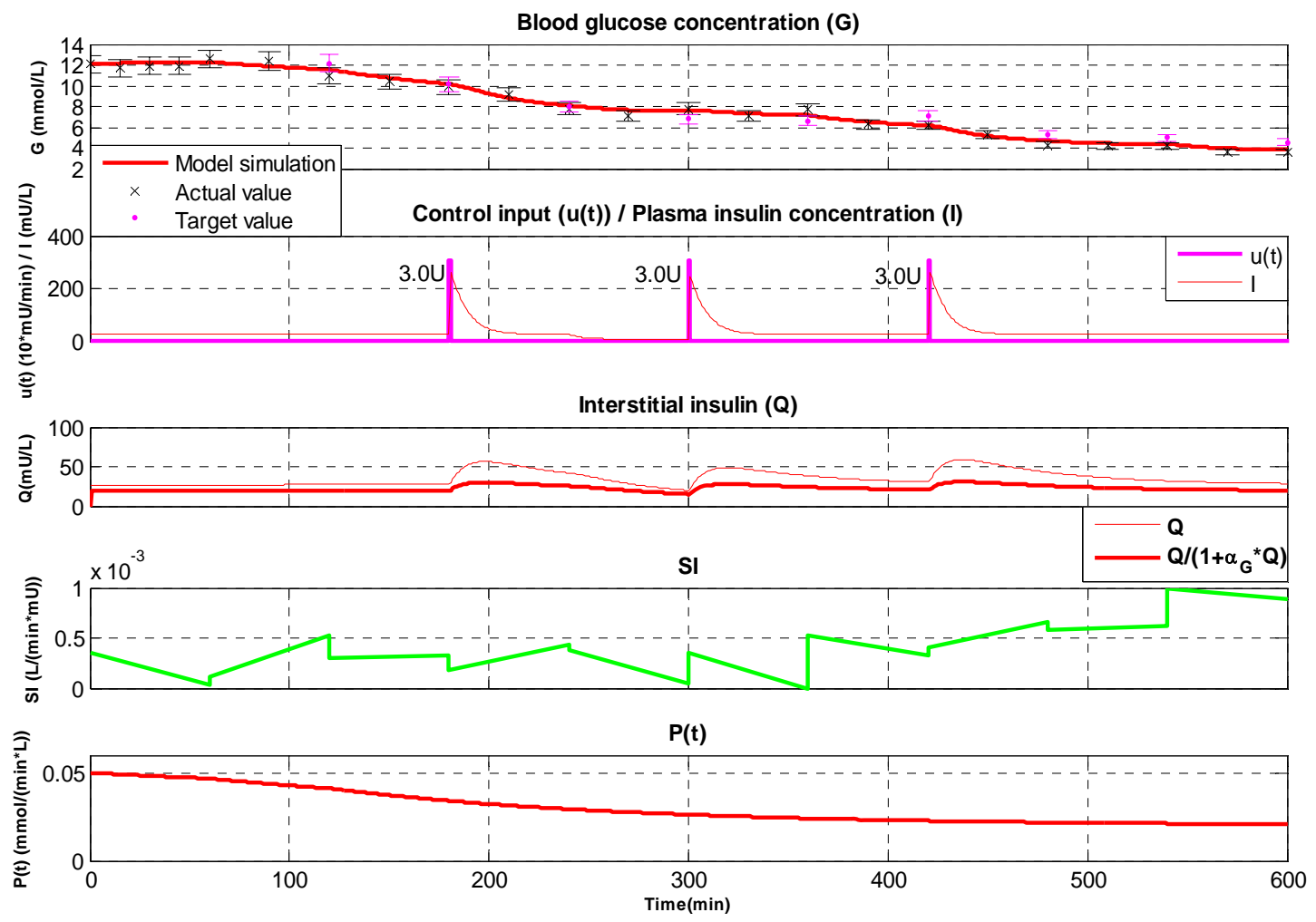

Figure 4: Patient 1 trial progression 

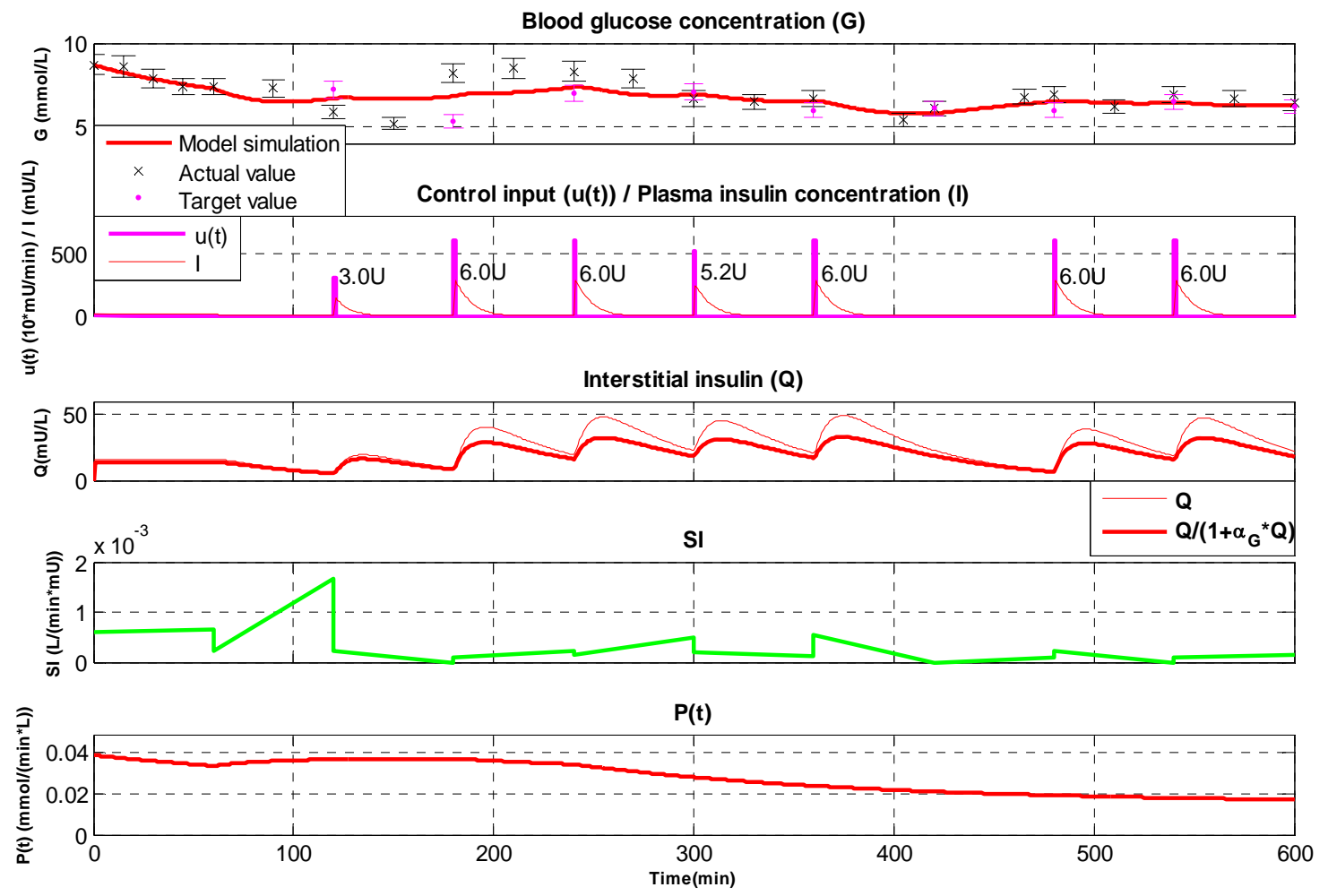

Figure 5: Patient 2 trial progression. A sudden change in patient condition occurred at 60120mins, resulting in heightened insulin resistance. 

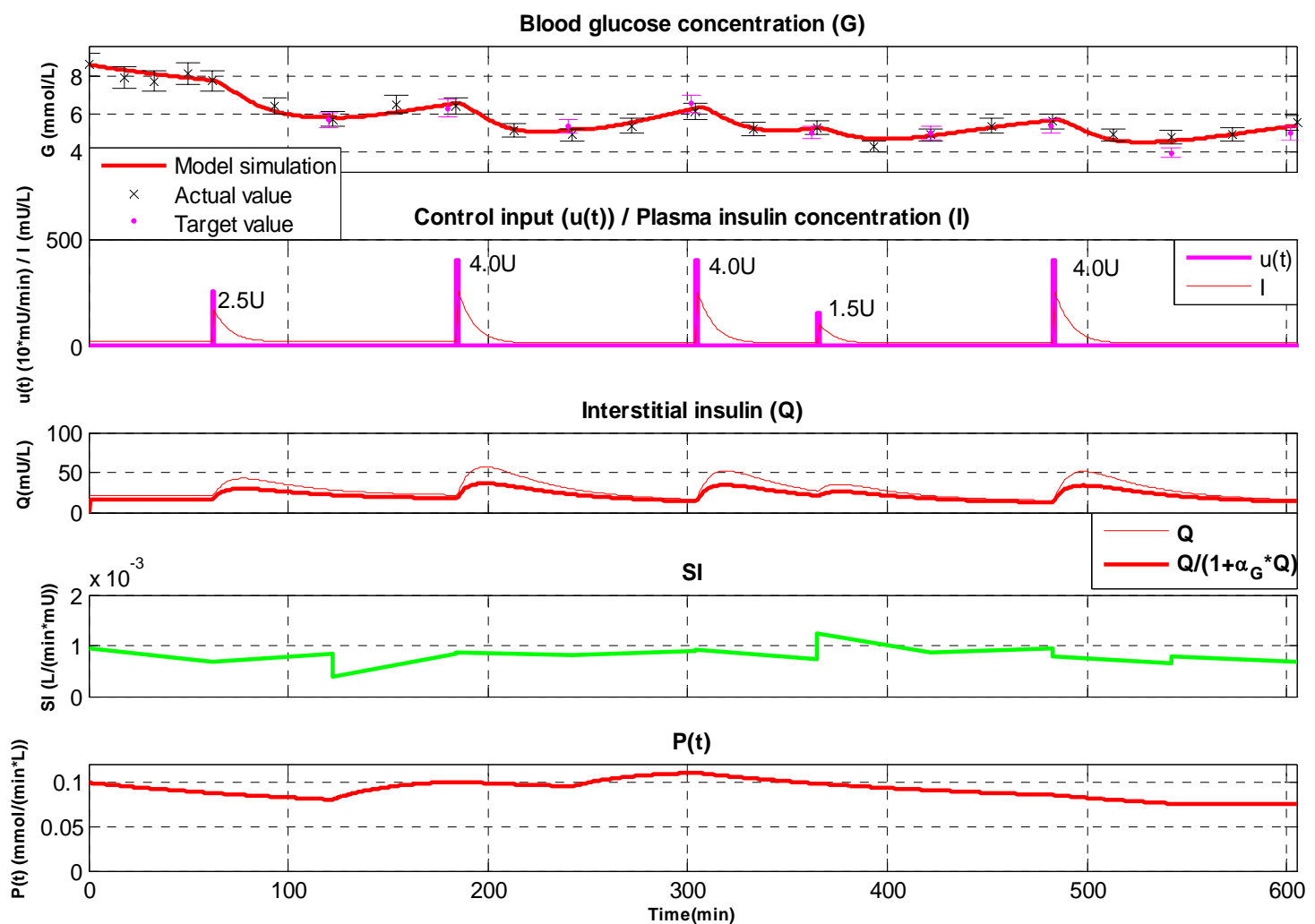

Figure 6: Patient 3 trial progression 

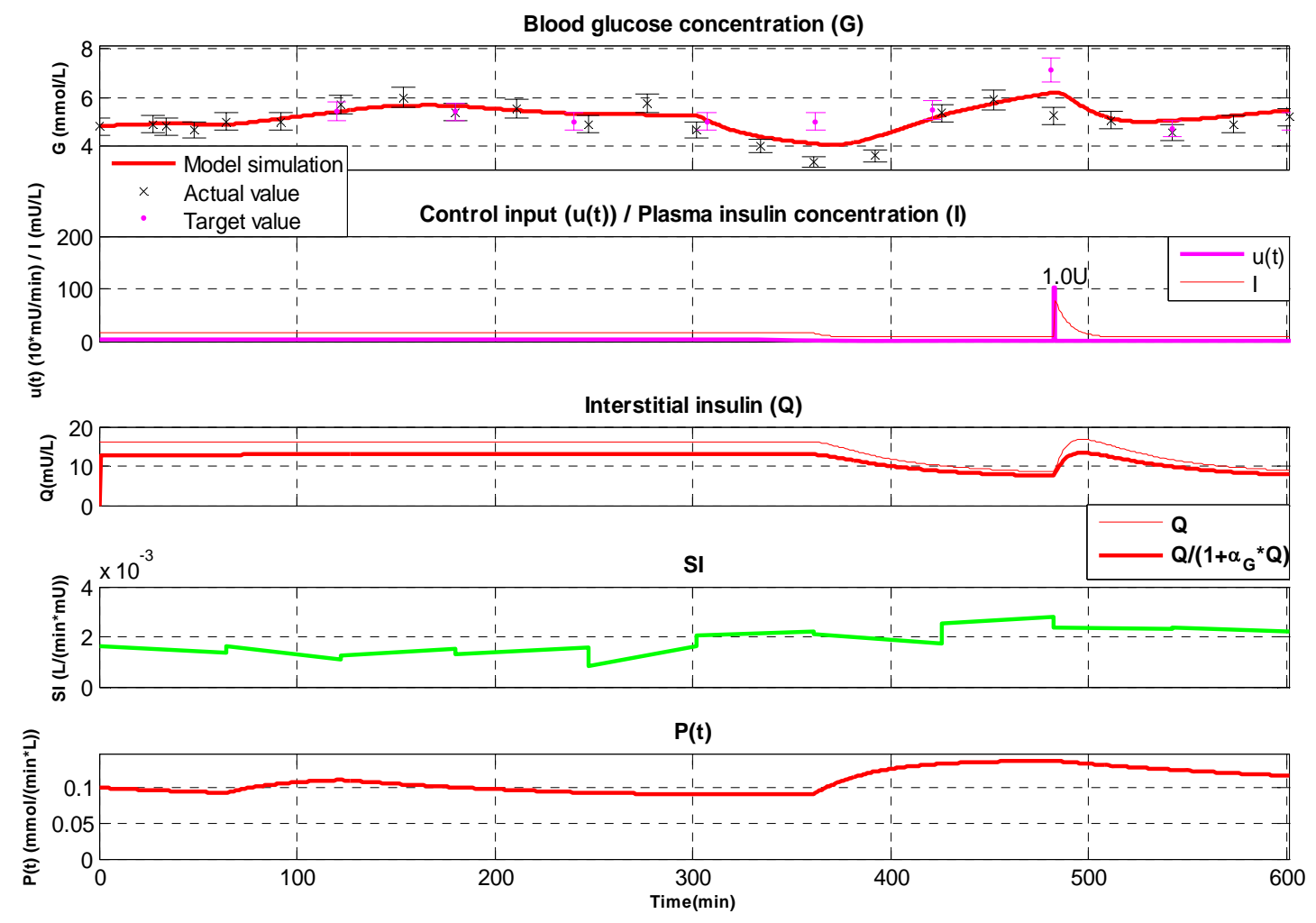

Figure 7: Patient 4 trial progression 


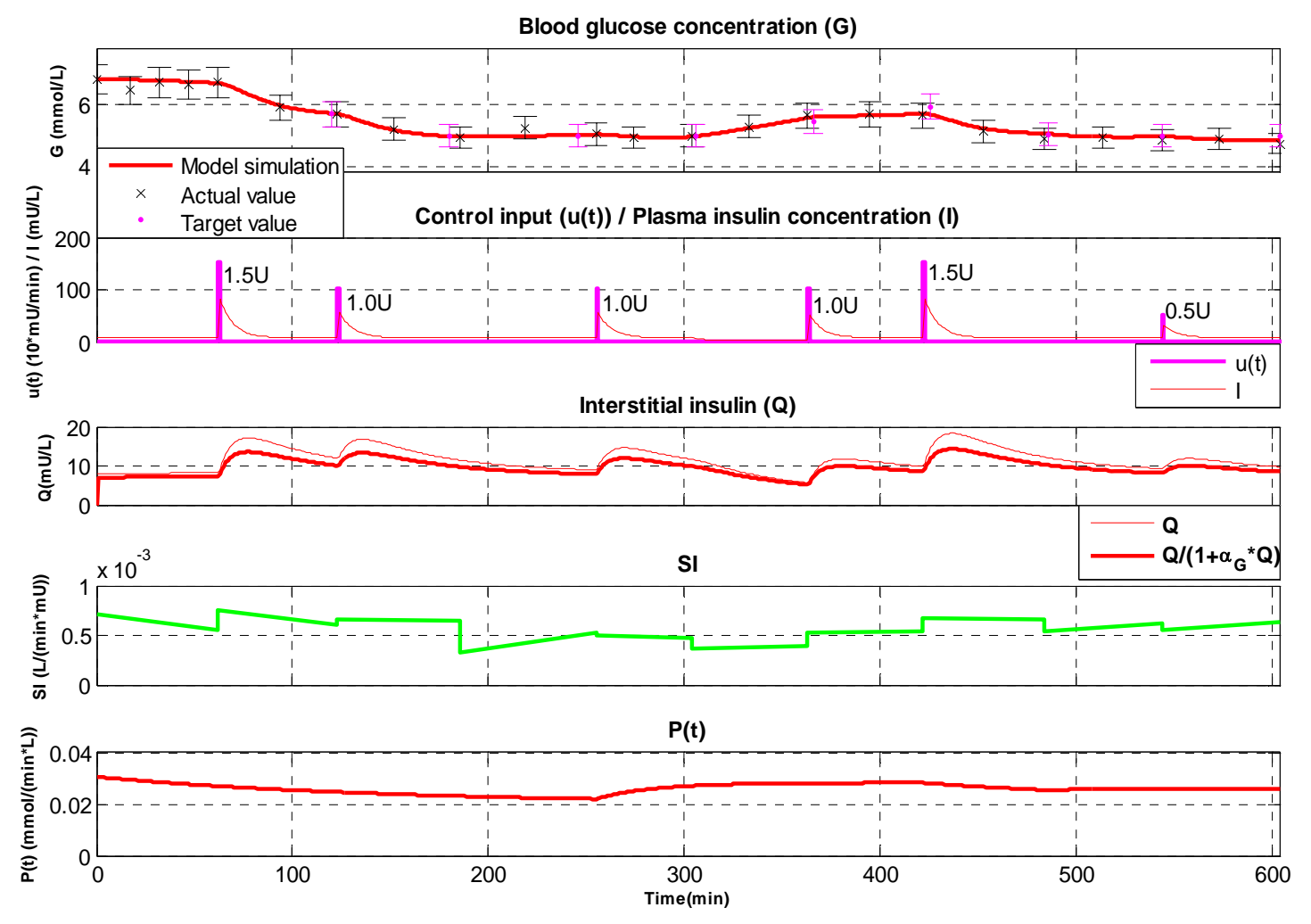

Figure 8: Patient 5 trial progression 
Blood glucose concentration (G)
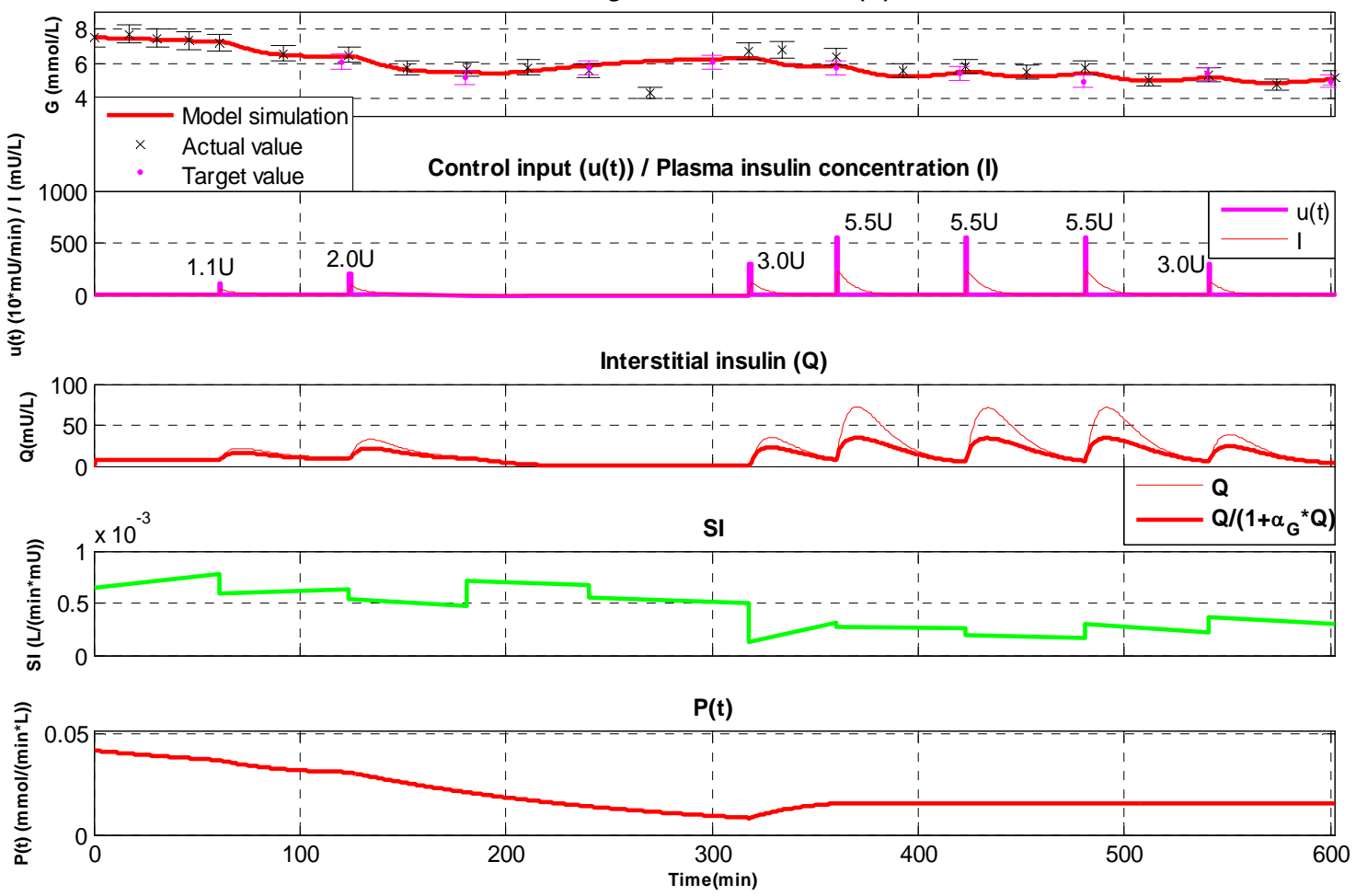

Figure 9: Patient 6 trial progression. Insulin resistance clearly increases at $\sim 320 \mathrm{mins}$ after MRI scan compared to 0-300mins. 

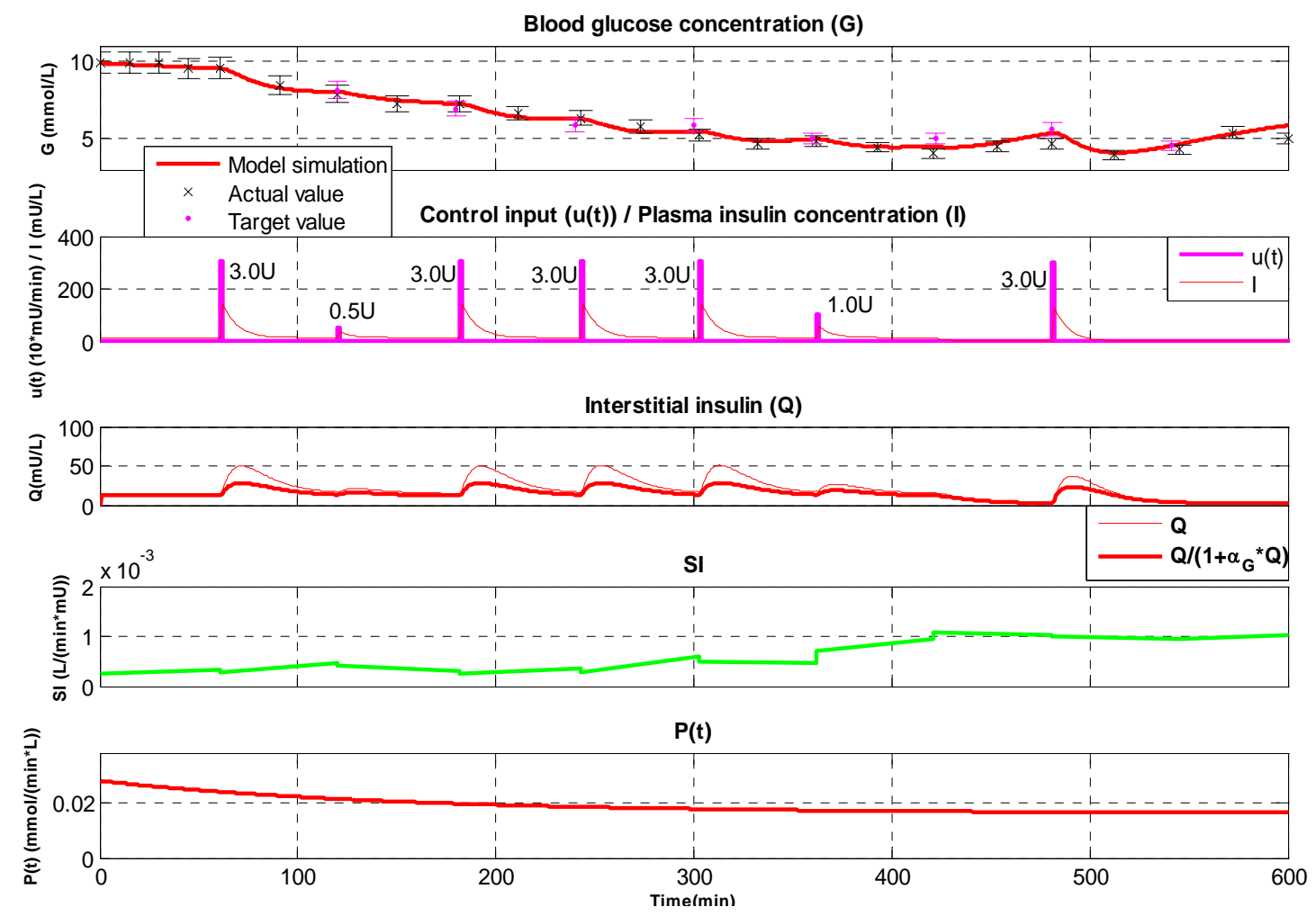

Figure 10: Patient 7 trial progression 

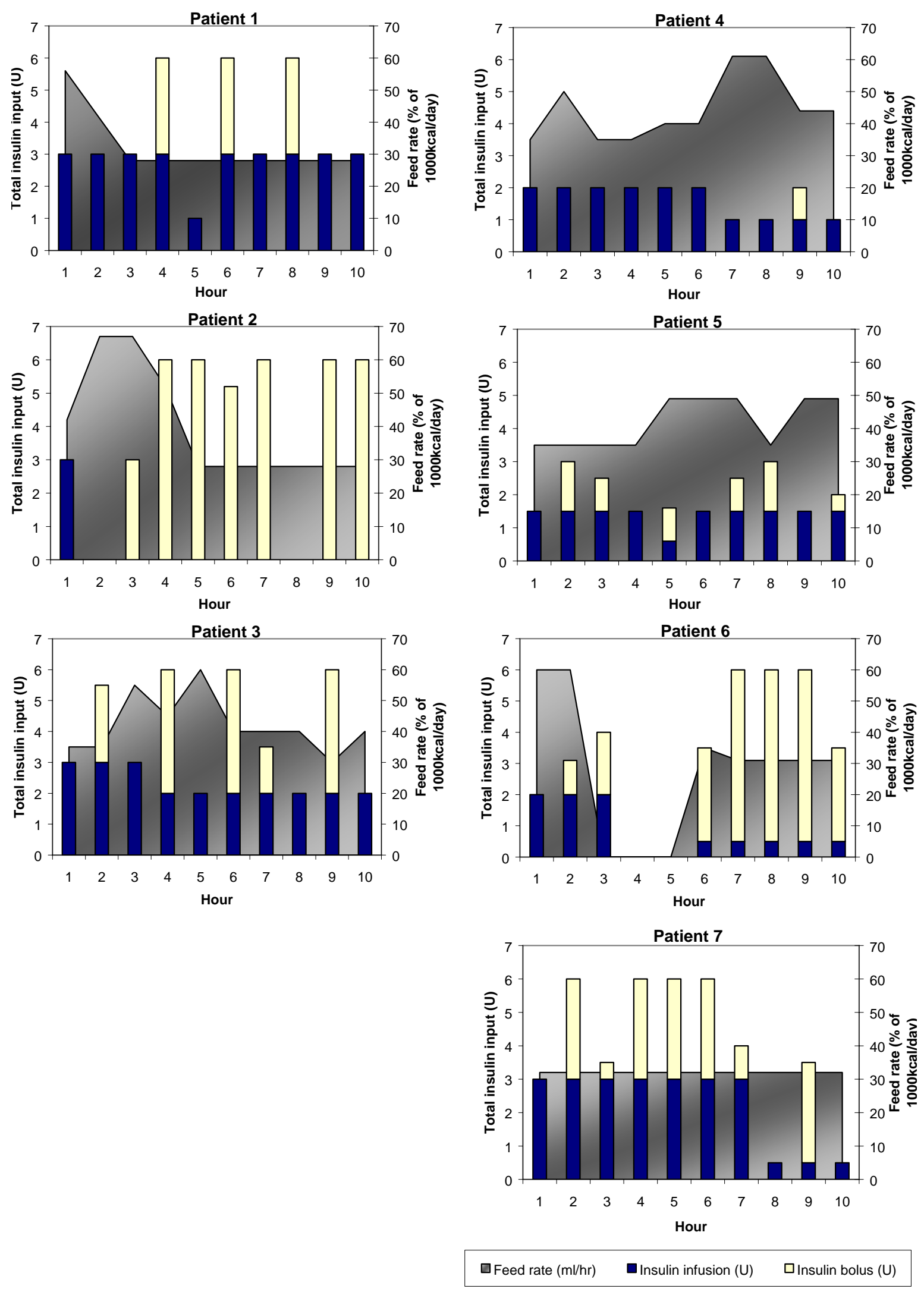

Figure 11: Clinical trial control input summary where maximum insulin input is 6U/hr and minimum feed rate is $31 \mathrm{ml} / \mathrm{hr}$ of RESOURCE ${ }^{\mathrm{TM}}$ Diabetic equivalent to $280 \mathrm{kcal} / \mathrm{day}$ of glucose 


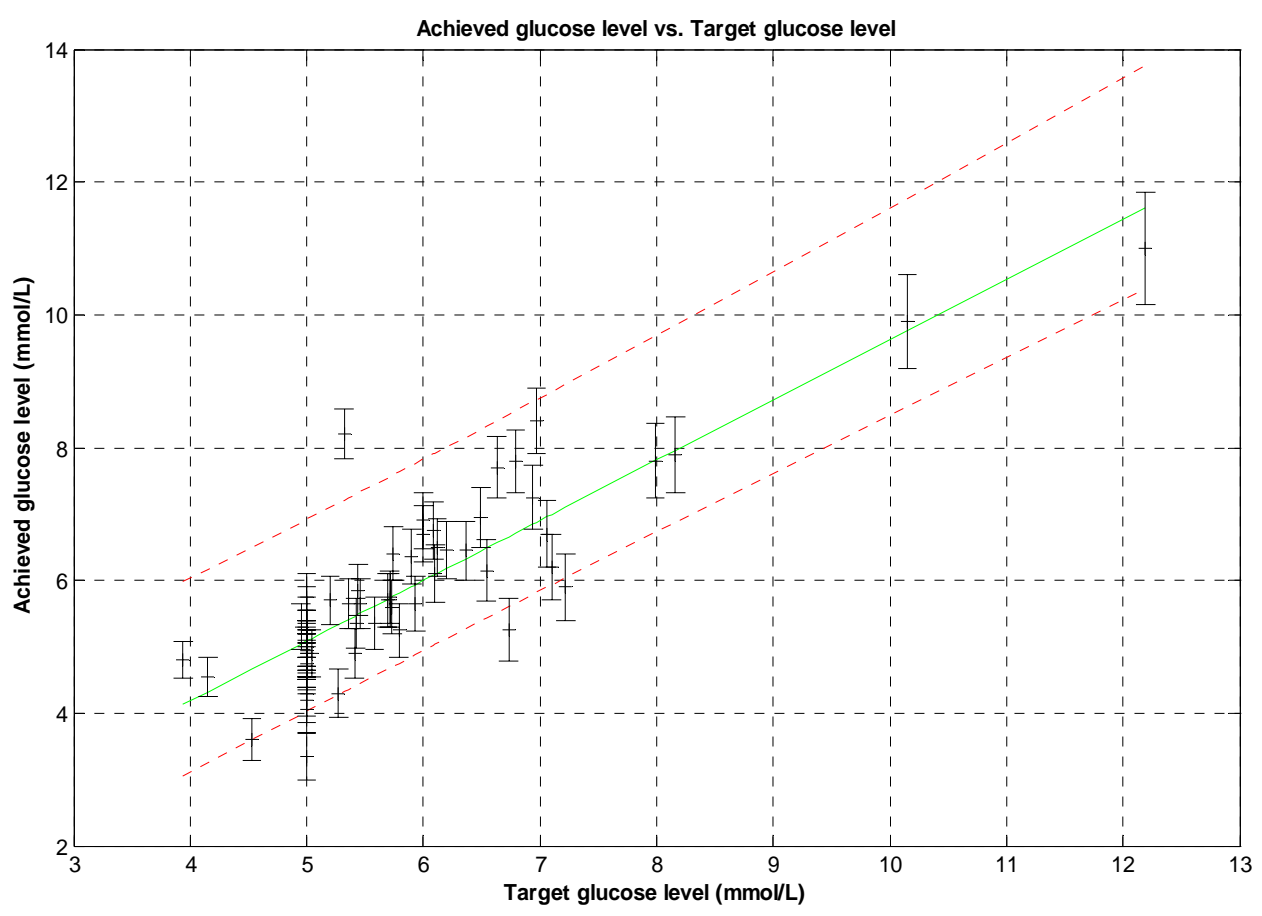

Figure 12: Achieved glucose level vs. target glucose level with $95 \%$ bootstrap confidence limits on estimation of achieved glucose level 


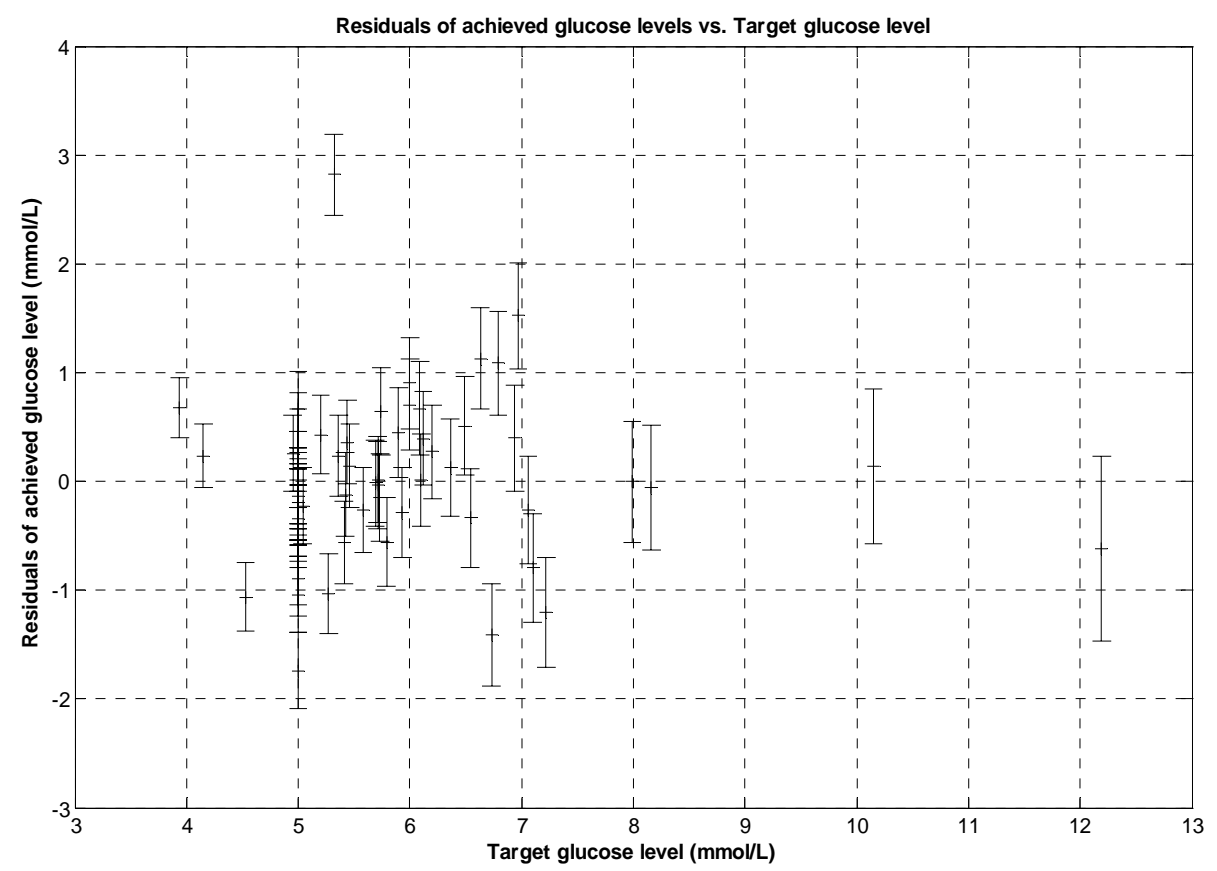

Figure 13: Plot of the residuals of the least squares linear model when applied to the achieved glucose level 


\section{TABLES}

Table 1: Patient cohort data

\begin{tabular}{clccccccccc}
\hline $\begin{array}{c}\text { Patient } \\
\text { number }\end{array}$ & $\begin{array}{c}\text { Medical } \\
\text { subgroup }\end{array}$ & $\begin{array}{c}\text { APACHE } \\
\text { II }\end{array}$ & $\begin{array}{c}\text { APACHE II } \\
\text { ROD }(\%)\end{array}$ & $\begin{array}{c}\text { APACHE } \\
\text { III }\end{array}$ & $\begin{array}{c}\text { SAPS } \\
\text { II }\end{array}$ & $\begin{array}{c}\text { SAPS II } \\
\text { ROD }(\%)\end{array}$ & Age & Sex & Mortality & Diabetes \\
\hline 1 & Sepsis & 17 & 14.3 & 40 & 15 & 2 & 56 & M & N & Type 2 \\
2 & Sepsis & 24 & 49.7 & 59 & 35 & 16.7 & 64 & M & N & \\
3 & Pulmonary & 31 & 73.3 & 85 & 45 & 34.8 & 60 & M & N & \\
4 & Sepsis & 26 & 59.7 & 91 & 62 & 71.9 & 75 & F & N & \\
5 & Sepsis & 21 & 33.2 & 58 & 34 & 15.3 & 73 & M & N & Type 2 \\
6 & Other medical & 17 & 14.3 & 44 & 44 & 32.6 & 57 & M & N & \\
7 & General surgical & 23 & 62.3 & 84 & 57 & 61.9 & 73 & F & N & Type 2 \\
\hline
\end{tabular}


Table 2: Clinical trial results summary

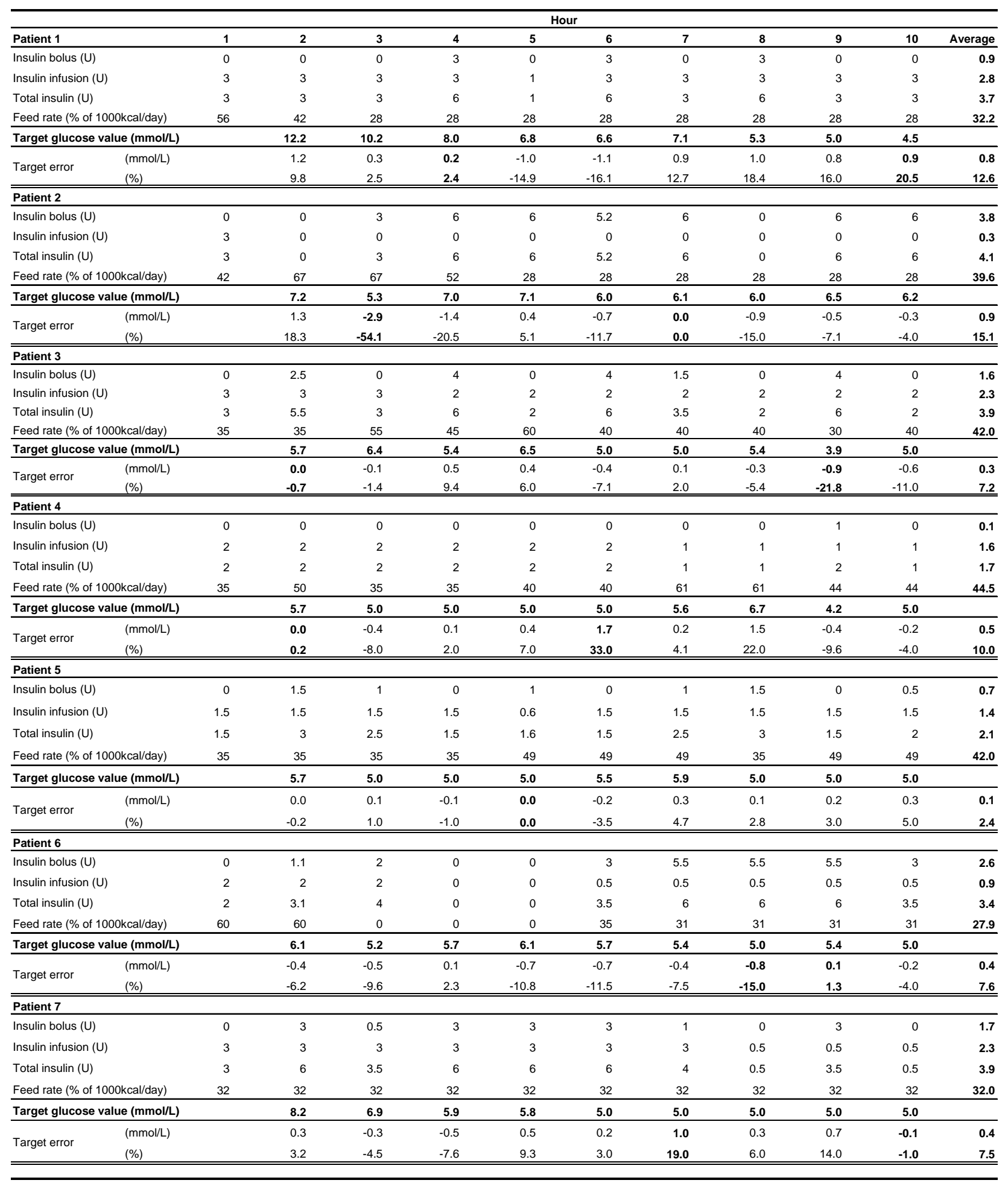

